\title{
BMJ Open The safety of intravitreal bevacizumab monotherapy in adult ophthalmic conditions: systematic review
}

\author{
Edith Poku, ${ }^{1}$ John Rathbone, ${ }^{2}$ Ruth Wong, ${ }^{1}$ Emma Everson-Hock, ${ }^{1}$ Munira Essat, ${ }^{1}$ \\ Abdullah Pandor, ${ }^{1}$ Allan Wailoo ${ }^{1}$
}

To cite: Poku E, Rathbone J, Wong $\mathrm{R}$, et al. The safety of intravitreal bevacizumab monotherapy in adult ophthalmic conditions: systematic review. BMJ Open 2014;4:e005244. doi:10.1136/bmjopen-2014005244

- Prepublication history and additional material is available. To view please visit the journal (http://dx.doi.org/ 10.1136/bmjopen-2014005244).

Received 11 March 2014 Revised 4 June 2014 Accepted 18 June 2014

CrossMark

\footnotetext{
${ }^{1}$ Health Economics and Decision Science, School of Health and Related Research, University of Sheffield, Sheffield, UK

${ }^{2}$ Faculty of Health Sciences and Medicine, Bond University, Gold Coast, Queensland, Australia

Correspondence to Dr Edith Poku; e.poku@sheffield.ac.uk
}

\section{ABSTRACT}

Objectives: To assess the safety of intravitreal bevacizumab (IVB) as a monotherapy and to evaluate the relationship between quality of treatment and adverse events.

Data sources: Cochrane Library, Ovid MEDLINE, MEDLINE in-process, Ovid EMBASE and Toxicology Literature Online (TOXLINE) from January 2009 to May 2012. Studies included in an earlier systematic review were also assessed for inclusion.

Study eligibility criteria, participants and interventions: Randomised controlled trials (RCTs), controlled trials or observational studies including $\geq 10$ participants reporting adverse events data following IVB monotherapy as a primary treatment in patients (aged 18 years or more) with any eye condition were included.

Study appraisal and synthesis methods: Study selection was undertaken independently by a minimum of two reviewers using pre-defined criteria. Data abstraction and quality assessment were performed by one reviewer, and then checked by a second reviewer. Study quality was assessed for only RCTs in accordance to the Cochrane Risk of Bias Tool. Additional items relating to safety data were also assessed. Results were tabulated or meta-analysed as appropriate.

Results: 22 RCTs and 67 observational studies were included. Only two RCTs reported valid safety data. Rates of serious adverse events following treatment were low. There was insufficient data to explore the relationship between the incidence of adverse events and quality of IVB injection.

Limitations: A majority of relevant existing studies were characterised by small sample sizes, unclear diagnostic criteria and reporting of safety outcomes. Conclusions and implications of key findings: Available evidence demonstrates low rates of serious local and systemic adverse events following treatment. However, the role of IVB quality in the incidence of adverse events remains unclear. Robust evidence is needed to examine the relationship between the incidence of adverse events and variables such as injection techniques, pre-existing risk factors (eg, immunosuppression, cross-contamination) and quality of IVB treatment.

\section{Strengths and limitations of this study}

Eighty-nine studies of bevacizumab monotherapy in patients with diverse ophthalmic conditions were included.

- A majority of relevant existing studies were characterised by small sample sizes, unclear diagnostic criteria and reporting of safety outcomes.

- The relationship between the incidence of adverse events and variables such as injection techniques, pre-existing risk factors (eg, immunosuppression, cross-contamination) and quality of bevacizumab could not be explored due to limited data.

\section{INTRODUCTION}

Age-related macular degeneration (AMD) and diabetic retinopathy (DR) have been identified as two of the three most common causes of age-specific visual impairment in England and Wales. ${ }^{1}$ More recently, effective treatment options have included anti-vascular endothelial growth factors (anti-VEGFs), which have been shown to both delay deterioration in vision as well as improve vision. ${ }^{2}$ Ranibizumab (Lucentis, Novartis) is licensed for the treatment of wet AMD and diabetic macular oedema (DMO) and costs $£ 742.17$ per injection $(0.23 \mathrm{~mL}$ vial $)$. Pegaptanib (Macugen; Pfizer) for treatment of AMD is available at a price of $£ 514$ per injection (300 $\mu \mathrm{g}$ vial). However, bevacizumab, which cost $£ 242.66$ for $4 \mathrm{~mL} / 100 \mathrm{mg}$ vial, is used as an unlicensed intervention in ophthalmic conditions. Although many doses for intravitreal administration can be produced from a single bevacizumab vial and therefore can be supplied for a much lower cost, the actual cost of dispensing smaller doses is uncertain. However, annual cost savings have been estimated if bevacizumab is used as standard treatment instead of ranibizumab in patients with AMD. ${ }^{3}$ 
Bevacizumab remains an unlicensed ophthalmic treatment for a number of reasons. There is an on-going debate with regard to intravitreal bevacizumab (IVB) use and its quality in clinical practice. One major concern relates to the risks associated with the reformulating the drug for intravitreal injections as well as possible adverse events (AEs) associated with systemically administered anti-VEGFs. Bevacizumab is reformulated for intravitreal use to deliver a smaller volume. However, the resulting reformulated product is considered by the Medicines and Healthcare products Regulatory Agency, a UK regulatory body for medicines and medical devices, as an unlicensed product. Another concern has centred predominantly on the possible risk of serious AEs such as endophthalmitis. To date, IVB safety evidence have been inconclusive. ${ }^{4}$ The aim of this review was to assess the safety, in terms of rates of specific serious AEs, of IVB monotherapy in ophthalmic conditions.

\section{METHODS}

We updated an existing systematic review on AEs of intravitreal anti-VEGF reported by van der Reis et al, ${ }^{5}$ which searched reports from 1948 to 2009 . We adapted the search strategy by including specific $\mathrm{AE}$ terms and omitting selected terms because the previous search strategy:

- included fewer AE terms

- used broad terms such as 'cause' and 'response' and

- applied specific study design filters, for example, in vitro studies.

Free text and subject headings or thesaurus terms relating to the intervention (eg, bevacizumab, avastin) were combined with $\mathrm{AE}$ floating subheadings or specific AE terms. We searched the Cochrane Library; Ovid MEDLINE; MEDLINE in-process; Ovid EMBASE; and Toxicology Literature Online (TOXLINE) from January 2009 to May 2012 because this review was part of a project commissioned by the National Institute for Health and Care Excellence (NICE) through its Decision Support Unit (DSU) between April to August 2012. We did not search clinical trial registers. No experimental and functional study design filter or language restrictions were used. Our MEDLINE search strategy presented as an on-line supplementary file 1 was translated across different databases. Reference lists of all relevant studies and systematic reviews were checked and a citation search of relevant articles was also undertaken.

\section{Study selection}

Study selection was undertaken independently by a minimum of two reviewers using pre-defined criteria. Any disagreements in the selection process were resolved by consensus or referral to a third reviewer. All published or unpublished randomised controlled trials (RCTs), controlled trials or observational studies including $\geq 10$ participants reporting $\mathrm{AE}$ data following IVB monotherapy as a primary treatment in patients (aged 18 years or more) with any eye condition were included. Relevant comparators were limited to monotherapies for RCTs only. Articles were excluded if patients had received prior treatment or received IVB as an adjunctive treatment. Non-English reports, narrative reviews, editorials, letters or publications relating to preclinical and biological studies were also excluded.

\section{Data extraction and quality assessment}

Data extraction and quality assessment were performed independently by one reviewer. Disagreements were resolved by discussion with a second reviewer and if agreement could not be reached, a third reviewer was consulted. Where multiple publications of the same study were identified, data were extracted and reported as a single study. Information abstracted included study characteristics, participant details (eg, number of patients, eye condition, mean age and baseline comparability), intervention and comparator details (eg, source, dose, injection quality and frequency of treatment) and outcomes. Outcomes of interest were limited to important and serious ocular and systemic AEs as listed below:

Systemic AEs

- Death

- Hospitalisation

- Non ocular haemorrhage (gastrointestinal, pulmonary, other non-ocular bleeds)

- Arterial thromboembolism

- Hypertension

- Myocardial infarction

- Cerebrovascular accident (stroke)

- Transient ischaemic attack

Ocular AEs

- Infectious endophthalmitis (infection of the eye)

- Retinal detachment

- Retinal (pigment epithelium) tear

- Anterior chamber reaction (including acute intraocular inflammation; uveitis; inflammation of the anterior chamber and hypopyon)

- Ocular haemorrhage

- Lens damage/injury (including cataract, clouding of the lens)

- Ocular hypertension (raised intraocular pressure $>21 \mathrm{~mm} \mathrm{Hg}$ )

- Visual loss

For RCTs, study quality was assessed in accordance to the Cochrane Risk of Bias Tool. Additionally, we assessed items relating to safety data for RCTs; these included follow-up period greater than 6 months, definition of $\mathrm{AE}$ and description of method of ascertaining AE. A formal quality assessment was not undertaken for observational studies. While checklists exist for evaluating the methodological quality of a range of non-randomised studies, there is no consensus on how to incorporate a single tool to appraise different study types in a review. ${ }^{6}$ It was anticipated that a variety of non-randomised study designs would be identified, so criteria assessed were 
limited to study design (eg, prospective or retrospective), length of follow-up and baseline comparability when appropriate.

\section{Data analysis}

A pooled analysis was undertaken using the Cochrane Review Manager software where appropriate. The relative risk was calculated for dichotomous outcomes using a fixed effects model (Mantel-Haenszel method). Otherwise, descriptive statistics were tabulated. Estimates of $\mathrm{AE}$ rates were calculated by dividing the number of events by the number of patients who received IVB (event rate per patient) or the number of eyes treated (event rate per treated eye).

\section{RESULTS}

A flow chart of the study selection is shown in figure 1 . Eighty-nine full text articles were included ( $\mathrm{n}=22 \mathrm{RCTs}$, $\mathrm{n}=67$ non-randomised studies). Of these 20 studies, including 1 RCT, were identified from the previous review. A total of 293 full text articles were excluded. Reasons for exclusion were wrong population, intervention or study type $(\mathrm{n}=162)$, unsuitable publication type (reviews, commentaries or editorials; $n=34$ ) and absence of usable data $(n=97)$. A full list of excluded studies with reasons for exclusion is available on request.

\section{Identified studies}

A total of 22 RCTs comparing IVB with a variety of interventions as well as an observational control group with safety data were included, as presented in table 1. ${ }^{2}{ }^{7-27}$ Study populations were patients with AMD ( $n=7$ studies); DMO ( $\mathrm{n}=8$ studies); retinal vein occlusion (RVO) ( $\mathrm{n}=4$ studies) and other ophthalmic conditions $(n=3)$. Assessment of study quality is presented as an on-line supplementary file 2. Study quality was considered to be moderate to low with only two RCTs ${ }^{16} 19$ meeting the criteria for valid safety data.

Sixty-seven observational studies were included as summarised in table 2. Most studies included patients with a single condition with fewer studies including a population with multiple conditions. Study quality was, generally, difficult to assess due to the quality of reporting. Approximately $65 \%$ of studies $(n=44 / 67)$ were retrospective in design with follow-up periods of more than 6 months reported in less than a third $(n=18 / 67)$ of included studies. Baseline characteristics of participants were comparable in two non-randomised studies ${ }^{28} 29$ and three case-control studies. ${ }^{30-32}$

\section{Treatment schedule and source}

Administration of $1.25 \mathrm{mg} / 0.05 \mathrm{~mL}$ was the most commonly reported dosage of IVB. Frequency of dosing and follow-up schedules varied across studies. Information relating to the source of IVB was reported in $35 \%$ $(n=14 / 22)$ of RCTs but less than a fifth $(19 \% ; n=13 / 67)$ of observational studies. ${ }^{28} \quad 42-44 \quad 4748 \quad 5962 \quad 66 \quad 68 \quad 74 \quad 8191$
IVB was mostly provided by a local dispensing service such as the hospital's pharmacy. There were limited data to assess quality of administered IVB.

\section{Reporting of AEs}

Ascertainment of AEs was presented more objectively in RCTs compared to observational studies. Non-RCT evidence was unclear because several studies reported absence of events as 'no serious complications'; or 'no ocular complications', or 'no adverse events were observed', thereby providing limited information on diagnostic techniques or criteria for reported AEs. Furthermore, for AEs such as visual loss, ocular haemorrhage, hypertension and hospitalisation, the relationship between the outcomes and treatment schedule or setting remained largely unclear.

\section{AEs reported in RCTs}

Pooled 1-year data ${ }^{16}{ }^{19}$ indicated that the risk of death (RR $1.38 ; 95 \%$ CI 0.71 to 2.68 ) or arteriothrombotic events (RR $0.81 ; 95 \%$ CI 0.42 to 1.59 ) were not significantly different between patients with AMD who received IVB or intravitreal ranibizumab (IVR). Furthermore, no significant difference in death between the IVB and IVR arms was observed when the CATT $^{16}$ (2 year data) and IVAN ${ }^{19}$ ( 1 year preliminary data) clinical trials were pooled to provide long-term data analyses as shown in figure 2. Cardiac disorders, transient ischaemic attack and hospitalisation for angina were not significantly different between patients with AMD treated with IVB and IVR. ${ }^{19}$ However, serious systemic AE rates remained significantly lower in the IVR group $(n=1795$, RR 1.27 CI 1.09 to 1.47).

Two smaller studies, Biswas et $a l^{15}$ and Gharbiya et $a l^{18}$ with safety data for patients with AMD reported no significant AEs. No significant differences were found for death and myocardial infarction (MI) in studies that compared IVB to pegaptinib ${ }^{26}$ or sham injection ${ }^{7}$ ( $\mathrm{n}=232$ patients, RR 0.30; 95\% CI 0.01 to 7.18).

Rates for endophthalmitis were not significantly different between IVB and IVR treatment groups for patients with AMD (1 RCT; RR 1.79; 95\% CI 0.53 to 6.08$).{ }^{16}$ There were no reports of endophthalmitis, ${ }^{9}{ }^{11}$ ocular hypertension, ${ }^{9}{ }^{11}$ retinal detachment ${ }^{2}{ }^{9}$ or vitreous haemorrhage $^{9}{ }^{11}$ in treatment groups comparing IVB with laser therapy in patients with DMO $(n=269)$. In patients with $\mathrm{DMO},{ }^{21} 22{ }^{25}$ ocular hypertension $(\mathrm{IOP}>21 \mathrm{~mm} \mathrm{Hg})$ was significantly higher in the IVT group ( $\mathrm{n}=183$; RR 0.13; CI 0.02 to 0.69) compared with the IVB group. A similar but non-significant trend was demonstrated in patients with $\mathrm{RVO}$ ( $\mathrm{n}=32$ patients; $\mathrm{RR}$ 0.08 ; CI 0.00 to 1.25$).{ }^{17}$

One short-term study at 3 months ${ }^{20}$ showed that posterior vitreous detachment was significantly higher in the IVB group compared with laser therapy $(n=110$; $R R$ 17.00; CI 1.01 to 287.50). However, the rates of uveitis, vitreous haemorrhage, pigment epithelial tears and cataract progression were low and indicated no significant 


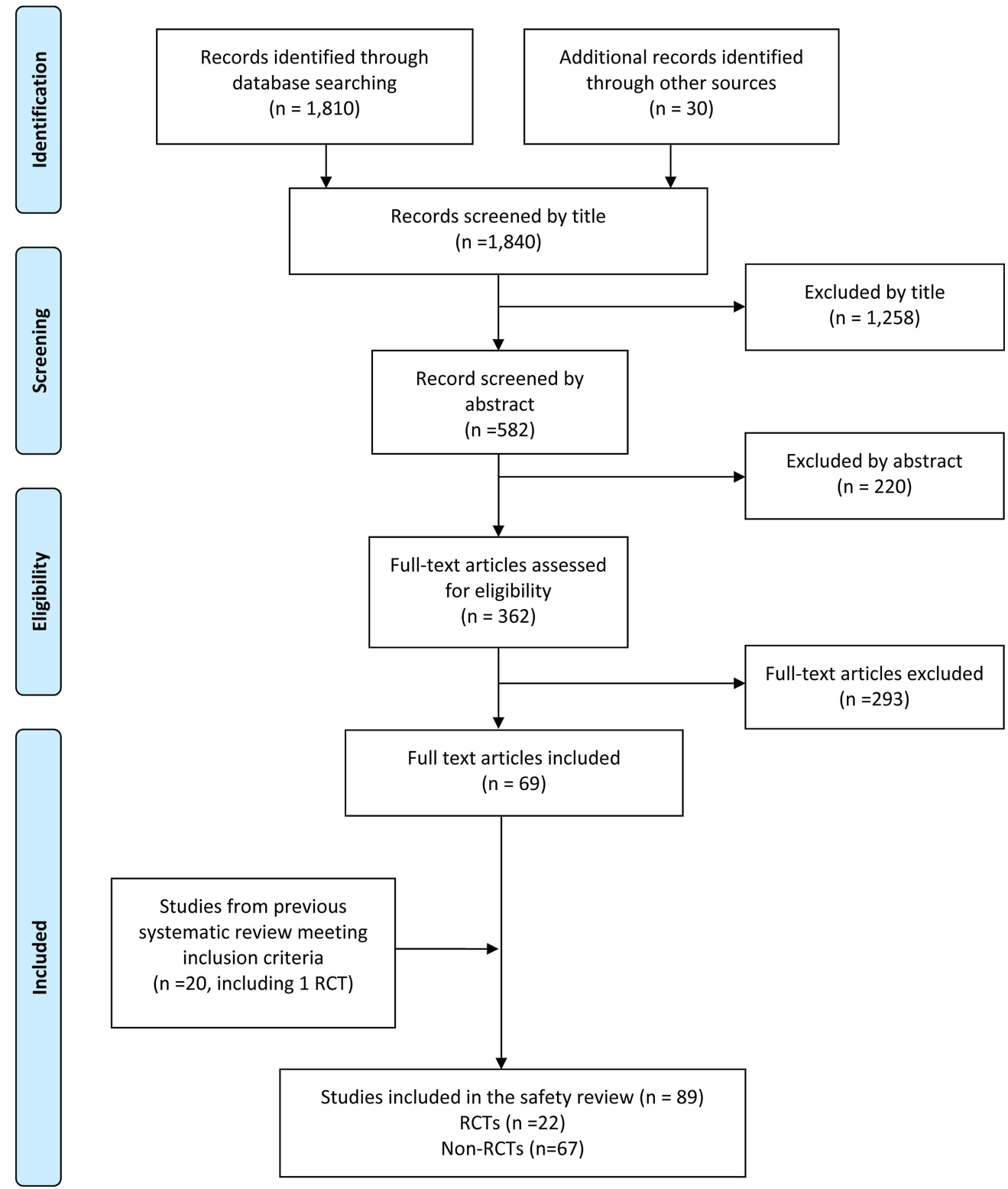

Figure 1 Summary of study selection. This flow chart outlines the process of study selection for the systemic review based on the recommendations of the PRISMA statement. RCT, randomised controlled trial.

differences between IVB and laser therapy. No significant differences in rates of foveal haemorrhage ${ }^{13}(n=81$; RR $0.62 ; 95 \%$ CI 0.28 to 1.35$)$ or hyphema ${ }^{27}$ ( $n=26$; RR 7.8 ; $95 \%$ CI 0.46 to 131.62) were found in patients with RVO who had IVB or sham injection.

\section{AEs reported in observational studies}

Table 3, summarising safety data reported in observational studies, displays extensive variation in the detail of reporting of AEs with most studies not reporting or observing AEs of interest. While high event rates were reported for hospitalisation, hypertension, anterior chamber reaction and visual loss, these rates need to be interpreted with caution due to previously mentioned issues with reporting along with likely confounders.
Systemic AEs reported included death (0.4-3.8\%), 40485494 arterial thromboembolism $(0-1.4 \%),{ }^{81}$ hypertension $(0-15.6 \%),{ }^{67} 778394$ MI $(0-8.2 \%),{ }^{28} 458194$ cerebrovascular accident $(0-8.7 \%)^{39} 45548394$ and transient ischaemic attack (TIA) $(0.4-1.0 \%) .{ }^{40} 81$

Visual loss was the most commonly reported ocular event, ${ }^{28} 32355053566989$ the definition of visual loss was often unclear and occasionally associated with AEs such as anterior chamber inflammation, severe intraocular inflammation or retinal detachment. Consequently, it is uncertain whether visual loss occurred as an AE from treatment or progression of the patient's condition. Infectious endophthalmitis was reported in 10 studies (range 0 $1.0 \%)$. Three of the 13 studies $^{2842-4447485962666874} 8191$ in which patients received locally prepared IVB reported cases of infectious endophthalmitis. Reported rates were 


\begin{tabular}{|c|c|c|c|c|c|c|}
\hline $\begin{array}{l}\text { Study identifier, } \\
\text { study location }\end{array}$ & $\begin{array}{l}\text { Description of study } \\
\text { population }\end{array}$ & $\begin{array}{l}\text { Number of } \\
\text { patients } \\
\text { (eyes) }\end{array}$ & $\begin{array}{l}\text { Interventions (treatment } \\
\text { schedule and numbers } \\
\text { treated) }\end{array}$ & $\begin{array}{l}\text { Comparators } \\
\text { (treatment schedule } \\
\text { and numbers treated) }\end{array}$ & $\begin{array}{l}\text { Reported safety } \\
\text { outcomes }\end{array}$ & $\begin{array}{l}\text { Information relating } \\
\text { to preparation of } \\
\text { intravitreal } \\
\text { bevacizumab }\end{array}$ \\
\hline \multicolumn{7}{|c|}{ Studies including patients with age-related macular degeneration ( $n=7$ studies) } \\
\hline $\begin{array}{l}\text { Bashshur et al, }{ }^{14} \\
\text { Lebanon }\end{array}$ & $\begin{array}{l}\text { Neovascular age-related } \\
\text { macular degeneration } \\
\text { BCVA, 20/50 to } 20 / 200 \\
\text { Submacular scarring or } \\
\text { haemorrhage, sparing the } \\
\text { fovea } \\
\text { Mean age, } 74.5 \text { years }\end{array}$ & $62(\mathrm{NR})$ & $\begin{array}{l}\text { IVB: } 2.5 \mathrm{mg} \text {, mean } 2.4 \\
\text { injections }(n=32)\end{array}$ & $\begin{array}{l}\text { Laser therapy: mean } \\
2.3 \text { sessions }(n=30)\end{array}$ & $\begin{array}{l}\text { Systemic adverse events } \\
\text { Hypertension } \\
\text { Outcomes at } 6 \text { months }\end{array}$ & $\begin{array}{l}\text { IVB prepared in hospital } \\
\text { pharmacy }\end{array}$ \\
\hline $\begin{array}{l}\text { Biswas et al, }{ }^{15} \\
\text { India }\end{array}$ & $\begin{array}{l}\text { Choroidal } \\
\text { neovascularisation } \\
\text { secondary to age-related } \\
\text { macular degeneration } \\
\text { BCVA, } 35 \text { to } 70 \text { ETDRS } \\
\text { letter } \\
\text { CMT, >250 } \mu \mathrm{m} \\
\text { Mean age, not reported }\end{array}$ & $60(60)$ & $\begin{array}{l}\text { IVB: } 1.25 \text { mg, } 3 \text { monthly } \\
\text { injections, mean } 4.3 \\
(n=30)\end{array}$ & $\begin{array}{l}\text { IVR: } 0.5 \mathrm{mg}, 3 \text { monthly } \\
\text { injections, mean } 5.6 \\
(n=30)\end{array}$ & $\begin{array}{l}\text { Significant adverse } \\
\text { events (unspecified) } \\
\text { Outcome at } 18 \text { months }\end{array}$ & $\begin{array}{l}\text { Methods of IVB } \\
\text { preparation not reported }\end{array}$ \\
\hline $\begin{array}{l}\text { CATT 2012, } \\
\text { USA }\end{array}$ & $\begin{array}{l}\text { Choroidal } \\
\text { neovascularisation } \\
\text { secondary to age-related } \\
\text { macular degeneration } \\
\text { BCVA, } 20 / 25 \text { to } 20 / 320 \\
\text { Mean age, } 79.5 \text { years }\end{array}$ & $1185(1107)$ & $\begin{array}{l}\text { IVB: } 1.25 \mathrm{mg} \text {, monthly or } \\
\text { as needed }(\mathrm{n}=586)\end{array}$ & $\begin{array}{l}\text { IVR: } 0.5 \text { mg, monthly } \\
\text { or as needed }(n=599)\end{array}$ & $\begin{array}{l}\text { Death } \\
\text { Endophthalmitis } \\
\text { Hypertension } \\
\text { Adverse events } \\
\text { associated with } \\
\text { anti-VEGF treatment } \\
\text { Arteriothrombotic adverse } \\
\text { events } \\
\text { Outcomes at } 2 \text { years }\end{array}$ & $\begin{array}{l}\text { Re-packaging of } \\
\text { commercially available } \\
\text { bevacizumab into glass } \\
\text { vials in an aseptic facility }\end{array}$ \\
\hline IVAN $2012,{ }^{19}$ UK & $\begin{array}{l}\text { Neovascular age-related } \\
\text { macular degeneration; } \\
\text { BCVA, } \geq 25 \text { ETDRS } \\
\text { letters } \\
\text { Mean age, } 77.0 \text { years }\end{array}$ & 610 & $\begin{array}{l}\text { IVB, } 1.25 \mathrm{mg} \text { as } \\
\text { continuous or as needed } \\
\text { treatment at } 3 \text { separate } \\
\text { visits }(n=296)\end{array}$ & $\begin{array}{l}\text { IVR, } 0.5 \mathrm{mg} \text { as } \\
\text { continuous or as } \\
\text { needed treatment at } 3 \\
\text { separate visits ( } n=314 \\
\text { eyes) }\end{array}$ & $\begin{array}{l}\text { Serious adverse events } \\
\text { Death } \\
\text { Arteriothrombotic events } \\
\text { Transient ischaemic } \\
\text { attack } \\
\text { Hospitalised for angina } \\
\text { Outcomes at } 1 \text { year }\end{array}$ & $\begin{array}{l}\text { Commercially } \\
\text { repackaged and prefilled } \\
\text { syringes IVB }\end{array}$ \\
\hline $\begin{array}{l}\text { Lazic and } \\
\text { Gabric, }{ }^{20} \text { Croatia }\end{array}$ & $\begin{array}{l}\text { Minimally classic or occult } \\
\text { choroidal } \\
\text { neovascularisation } \\
\text { secondary to age-related } \\
\text { macular degeneration } \\
\text { BCVA, } \geq 20 / 400 \\
\text { Mean age, } 75.7 \text { years }\end{array}$ & $165(165)$ & IVB, $1.25 \mathrm{mg}(\mathrm{n}=55)$ & $\begin{array}{l}\text { 1. Laser therapy: } \\
\text { according to } \\
\text { recommended } \\
\text { standard procedures } \\
(\mathrm{n}=55) \\
2 . \text { Combination } \\
\text { treatment, that is, laser }\end{array}$ & $\begin{array}{l}\text { Pigment epithelial tears } \\
\text { Posterior vitreous } \\
\text { detachment } \\
\text { Thromboembolic events } \\
\text { Cataract progression } \\
\text { Outcomes at } 3 \text { months }\end{array}$ & $\begin{array}{l}\text { Methods of IVB } \\
\text { preparation not reported }\end{array}$ \\
\hline
\end{tabular}


Study identifier, Description of study study location population
Number of Interventions (treatment Comparators patients

(eyes)

\section{schedule and numbers}

treated)
Schimid-Kubista et $a l,{ }^{24}$ Austria

Tufail et $a{ }^{26}$ UK
Choroidal

secondary to neovascular

age-related macular

degeneration

BCVA, 5 to 40 ETDRS

letters

Mean age, 77.5 years

Neovascular age-related

macular degeneration;

BCVA, $6 / 12$ to $6 / 96$

(Snellen

equivalent) or 25 to 70

ETDRS letter scores

Mean age, 81 years neovascularisation
$48(48)$

IVB, $1.0 \mathrm{mg}$ every

6 weeks; total of 3 injections $(n=13)$

31 (NR)

IVB, $1.25 \mathrm{mg}$, three loading injections at 6 week intervals followed by further treatment if required at 6 week $7.1(n=65)$.
6 weeks; total of 3

Outcomes at 1 year

Studies including patients with diabetic macular oedema ( $n=8$ studies)

Ahmadieh et al, ${ }^{7} \quad$ Diabetic macular oedema BCVA, $\leq 20 / 40$

Mean age, 59.7 years

$101(115)$

DRCRN 2007, ${ }^{10}$ Diabetic macular oedema USA (patients with type 1 and type 2 diabetes) BCVA, ETDRS VA letter score, 24 to78 (20/32 to 20/320

$\mathrm{CMT} \geq 275 \mu \mathrm{m}$

Median age, 65 years
1. IVB, $1.25 \mathrm{mg}$ and IVT, 2 mg at baseline, then IVB, $1.25 \mathrm{mg}$ at weeks 6 and $12(n=37$ eyes)

2. Sham injection $(n=37$ eyes)

1. Laser treatment at baseline ( $\mathrm{n}=19$ eyes) 2. IVB, $1.25 \mathrm{mg}$ at baseline, laser

treatment at week 3 then IVB, $1.25 \mathrm{mg}$ at week 6 ( $n=22$ eyes)

3. IVB, $1.25 \mathrm{mg}$ at

baseline, sham at week 6 ( $n=22$ eyes)
Reported safety

therapy followed by

IVB, $1.25 \mathrm{mg}$ within an hour $(\mathrm{n}=55)$ intervals, mean injections
IVP $0.3 \mathrm{mg}$ every

injections $(n=18)$

2. IVB, $1.0 \mathrm{mg}$ then two injections of IVP $0.3 \mathrm{mg} 6$ weeks apart $(n=17)$

1. Laser therapy,

$(n=16)$

2. IVP, $0.3 \mathrm{mg},(n=38)$

3. Sham injection $(n=12)$
IOP

Raised blood pressure

Outcomes at 6 months

Endophthalmitis

Uveitis

Retinal detachment

Retinal tear

Vitreous haemorrhage

Lens damage

Myocardial infarction

Stroke

Cerebral infarction

Death

IVB, $1.25 \mathrm{mg}$ at baseline and weeks 6 and 12 ( $n=41$ eyes)

Information relating

to preparation of

intravitreal

bevacizumab

Methods of IVB

preparation not reported

Death

Marked anterior chamber

reaction

Progression of fibrous

proliferation

Outcomes at 24 weeks

Endophthalmitis, raised intraocular pressure,

raised blood pressure,

myocardial infarction,

2. IVB, $2.5 \mathrm{mg}$

congestive heart failure

baseline, week $6(n=24$

Outcomes over a

70-week period
IVB injections were prepared as single use syringes with a shelf life of 6 weeks. Syringes were placed in sealed plastic pouches

Methods of IVB

preparation not reported

Methods of IVB

preparation not reported 


\begin{tabular}{|c|c|c|c|c|c|c|}
\hline $\begin{array}{l}\text { Study identifier, } \\
\text { study location }\end{array}$ & $\begin{array}{l}\text { Description of study } \\
\text { population }\end{array}$ & $\begin{array}{l}\text { Number of } \\
\text { patients } \\
\text { (eyes) }\end{array}$ & $\begin{array}{l}\text { Interventions (treatment } \\
\text { schedule and numbers } \\
\text { treated) }\end{array}$ & $\begin{array}{l}\text { Comparators } \\
\text { (treatment schedule } \\
\text { and numbers treated) }\end{array}$ & $\begin{array}{l}\text { Reported safety } \\
\text { outcomes }\end{array}$ & $\begin{array}{l}\text { Information relating } \\
\text { to preparation of } \\
\text { intravitreal } \\
\text { bevacizumab }\end{array}$ \\
\hline $\begin{array}{l}\text { Faghihi et al, }{ }^{11} \\
\text { Iran }\end{array}$ & $\begin{array}{l}\text { Diabetic macular oedema } \\
\text { (patients with type } 2 \\
\text { diabetes) } \\
\text { BCVA, } \leq 20 / 40 \\
\text { CMT, >250 } \mu \mathrm{m} \\
\text { Mean age, } 57.5 \text { years }\end{array}$ & $110(130)$ & $\begin{array}{l}\text { IVB, } 1.25 \mathrm{mg} \text {, dosing } \\
\text { schedule not reported ( } \mathrm{n} \\
=42 \text { eyes) }\end{array}$ & $\begin{array}{l}\text { 1. Laser }(n=47 \text { eyes }) \\
\text { 2. IVB }(1.25 \mathrm{mg})+\text { IVT } \\
(2 \mathrm{mg})(\mathrm{n}=41 \text { eyes })\end{array}$ & $\begin{array}{l}\text { Safety assessment } \\
\text { Vitreous haemorrhage } \\
\text { Ocular hypertension } \\
\text { ( } \geq 23 \mathrm{~mm} \mathrm{Hg} \text { ) } \\
\text { Outcome } 16 \text { weeks }\end{array}$ & $\begin{array}{l}\text { Methods of IVB } \\
\text { preparation not reported }\end{array}$ \\
\hline Lim et al, ${ }^{21}$ Korea & $\begin{array}{l}\text { Diabetic macular oedema } \\
\text { BCVA, not reported } \\
\text { CMT } \geq 300 \mu \mathrm{m} \\
\text { Mean age, } 60.0 \text { years }\end{array}$ & $111(120)$ & $\begin{array}{l}\text { IVB, } 1.25 \mathrm{mg} \text { at baseline } \\
\text { and at week } 6(n=36)\end{array}$ & $\begin{array}{l}\text { 1. IVT, } 2 \mathrm{mg}(\mathrm{n}=38) \\
\text { 2. IVB }(1.25 \mathrm{mg})+\mathrm{IVT} \\
(2 \mathrm{mg})(\mathrm{n}=37)\end{array}$ & $\begin{array}{l}\text { Hypertension } \\
\text { Thromboembolic AE } \\
\text { Serious ocular } \\
\text { complications } \\
\text { IOP } \\
\text { Outcomes at } 1 \text { year }\end{array}$ & $\begin{array}{l}\text { Methods of IVB } \\
\text { preparation not reported }\end{array}$ \\
\hline $\begin{array}{l}\text { Marey and } \\
\text { Ellakwa, }{ }^{22} \text { Egypt }\end{array}$ & $\begin{array}{l}\text { Clinically significant } \\
\text { diabetic macular oedema } \\
\text { BCVA, not reported } \\
\text { CMT at baseline, reported } \\
\text { per study groups } \\
\text { Mean age, } 57.7 \text { years }\end{array}$ & $90(90)$ & IVB, $1.25 \mathrm{mg}(\mathrm{n}=30)$ & $\begin{array}{l}\text { 1. IVT, } 4 \mathrm{mg}(\mathrm{n}=30) \\
\text { 2. IVB }(1.25 \mathrm{mg})+\mathrm{IVT} \\
(4 \mathrm{mg})(\mathrm{n}=30)\end{array}$ & $\begin{array}{l}\text { IOP }>22 \mathrm{~mm} \mathrm{Hg} \\
\text { Cataracts } \\
\text { Outcomes at } 12 \text { weeks }\end{array}$ & $\begin{array}{l}\text { Methods of IVB } \\
\text { preparation not reported }\end{array}$ \\
\hline $\begin{array}{l}\text { Michaelides } \\
\text { et } a l,{ }^{9} \text { UK }\end{array}$ & $\begin{array}{l}\text { Clinically significant } \\
\text { diabetic macular oedema } \\
\text { (in patients with type } 1 \text { or } \\
\text { type } 2 \text { diabetes) } \\
\text { BCVA, } 35 \text { to } 69 \text { ETDRS } \\
\text { letters } \\
\text { CMT } \geq 279 \mu \mathrm{m} \\
\text { Mean age, } 64.2 \text { years }\end{array}$ & $80(80)$ & $\begin{array}{l}\text { IVB, } 1.25 \mathrm{mg} \text { at baseline, } \\
\text { and then every } 6 \text { weeks } \\
\text { as needed; number of } \\
\text { injections ranged } \\
\text { between } 3 \text { and } 9(n=42)\end{array}$ & $\begin{array}{l}\text { Laser therapy, every } \\
4 \text { months as needed; } \\
\text { number of treatments, } \\
\text { ranged between } 1 \text { and } \\
4(n=38)\end{array}$ & $\begin{array}{l}\text { Death } \\
\text { IOP } \\
\text { Loss of } 30 \text { ETDRS letters } \\
\text { Vitreous haemorrhage } \\
\text { Cerebrovascular accident } \\
\text { Outcomes at } 12 \text { months }\end{array}$ & $\begin{array}{l}\text { IVB prepared by } \\
\text { Moorfields, London }\end{array}$ \\
\hline $\begin{array}{l}\text { Shahin et al, }{ }^{25} \\
\text { Egypt }\end{array}$ & $\begin{array}{l}\text { Diffuse diabetic macular } \\
\text { oedema } \\
\text { BCVA, not reported } \\
\text { CMT } \geq 292 \mu \mathrm{m} \\
\text { Mean age, } 52.7 \text { years }\end{array}$ & $32(48)$ & $\begin{array}{l}\text { IVB, } 1.25 \mathrm{mg} \text {, single } \\
\text { injection ( } \mathrm{n}=24 \text { eyes) }\end{array}$ & $\begin{array}{l}\text { IVT, } 4 \text { mg, single } \\
\text { injection ( } n=24 \text { eyes) }\end{array}$ & $\begin{array}{l}\text { IOP }(\geq 23-43 \mathrm{~mm} \mathrm{Hg}) \\
\text { Visually significant } \\
\text { cataract } \\
\text { Outcomes at } 3 \text { months }\end{array}$ & $\begin{array}{l}\text { Methods of IVB } \\
\text { preparation not reported }\end{array}$ \\
\hline $\begin{array}{l}\text { Soheilian et al, }{ }^{2} \\
\text { Iran }\end{array}$ & $\begin{array}{l}\text { Diabetic macular oedema } \\
\text { BCVA, } 20 / 40 \text { to } 20 / 300 \\
\text { CMT, not used as } \\
\text { inclusion criterion } \\
\text { Mean age, } 60.5 \text { years }\end{array}$ & $129(150)$ & $\begin{array}{l}\text { IVB, } 1.25 \mathrm{mg} \text { at baseline; } \\
\text { treated repeated at } 3 \\
\text { monthly interval on an } \\
\text { as-needed basis ( } \mathrm{n}=50 \\
\text { eyes) }\end{array}$ & $\begin{array}{l}\text { 1. IVB, } 1.25 \mathrm{mg}+\mathrm{IVT} \text {, } \\
2 \mathrm{mg} \text {; treated repeated } \\
\text { at } 3 \text { monthly interval } \\
\text { on an as-needed basis } \\
(\mathrm{n}=50 \text { eyes) }\end{array}$ & $\begin{array}{l}\text { Death } \\
\text { Lens opacities } \\
\text { IOP } \\
\text { Vitreous haemorrhage } \\
\text { High risk proliferative }\end{array}$ & $\begin{array}{l}\text { Methods of IVB } \\
\text { preparation not reported }\end{array}$ \\
\hline
\end{tabular}


Studies including patients with retinal vein occlusion ( $n=4$ studies)

\begin{tabular}{|c|c|c|c|c|c|c|}
\hline study location & population & (eyes) & treated) & and numbers treated) & outcomes & bevacizumab \\
\hline \multicolumn{7}{|c|}{ Studies including patients with retinal vein occlusion ( $n=4$ studies) } \\
\hline $\begin{array}{l}\text { Cekic et al, } \\
\text { Turkey }\end{array}$ & $\begin{array}{l}\text { Macular oedema due to } \\
\text { branch retinal vein } \\
\text { occlusion } \\
\text { BCVA, } \leq 20 / 40 \\
\text { CMT, }>250 \mu \mathrm{m} \\
\text { Mean age, } 63 \text { years }\end{array}$ & $21(21)^{*}$ & $\begin{array}{l}\text { IVB: } 1.25 \mathrm{mg} \text {, mean } 1.6 \\
\text { injections } \\
(\mathrm{n}=14)\end{array}$ & $\begin{array}{l}\text { IVT: } 4 \mathrm{mg} \text {, mean } 1.4 \\
\text { injections }(\mathrm{n}=17) \\
\text { IVT+IVB }(\mathrm{n}=21)\end{array}$ & $\begin{array}{l}\text { Endophthalmitis, uveitis, } \\
\text { thromboembolic events } \\
\text { Outcomes at } 6 \text { months }\end{array}$ & $\begin{array}{l}\text { Methods of IVB } \\
\text { preparation not reported }\end{array}$ \\
\hline $\begin{array}{l}\text { Ding et al, }{ }^{17} \\
\text { China }\end{array}$ & $\begin{array}{l}\text { Macular oedema } \\
\text { secondary to retinal vein } \\
\text { occlusion (unspecified) } \\
\text { BCVA, } \leq 20 / 40 \\
\text { CMT, }>250 \mu \mathrm{m} \\
\text { Mean age, } 54 \text { years }\end{array}$ & $31(32)$ & $\begin{array}{l}\text { IVB, } 1.25 \mathrm{mg} \text {, repeat } \\
\text { treatment given if } \\
\text { condition persisted or } \\
\text { recurred ( } n=16 \text { eyes) }\end{array}$ & $\begin{array}{l}\text { IVT, } 4 \text { mg, repeat } \\
\text { treatment given if } \\
\text { condition persisted or } \\
\text { recurred ( } n=16 \text { eyes) }\end{array}$ & $\begin{array}{l}\text { IOP }>21 \mathrm{~mm} \mathrm{Hg} \\
\text { Outcomes at } 9 \text { months }\end{array}$ & $\begin{array}{l}\text { Methods of IVB } \\
\text { preparation not reported }\end{array}$ \\
\hline $\begin{array}{l}\text { Epstein et al, }{ }^{12} \\
\text { Sweden }\end{array}$ & $\begin{array}{l}\text { Macular oedema } \\
\text { secondary to central } \\
\text { retinal vein occlusion } \\
\text { BCVA, } 15 \text { to } 65 \text { ETDRS } \\
\text { letters (approx. } 20 / 50 \text { to } \\
20 / 500 \text { ) } \\
\text { CMT } \geq 300 \mu \mathrm{m}\end{array}$ & $60(60)$ & $\begin{array}{l}\text { IVB, } 1.25 \mathrm{mg} \text { at baseline } \\
\text { and at weeks } 6,12 \text { and } \\
18(n=30)\end{array}$ & $\begin{array}{l}\text { Sham injection: at } \\
\text { baseline and at weeks } \\
6,12 \text { and } 18(n=30)\end{array}$ & $\begin{array}{l}\text { Endophthalmitis } \\
\text { Retinal tear } \\
\text { Retinal detachment } \\
\text { No serious non-ocular } \\
\text { adverse events } \\
\text { Outcomes at } 6 \text { months }\end{array}$ & $\begin{array}{l}\text { IVB prepared in hospital } \\
\text { pharmacy }\end{array}$ \\
\hline $\begin{array}{l}\text { Moradian et al, }{ }^{13} \\
\text { Iran }\end{array}$ & $\begin{array}{l}\text { Acute branch retinal vein } \\
\text { occlusion } \\
\text { BCVA, } \leq 20 / 50 \\
\text { Mean age, } 57.6 \text { years }\end{array}$ & $81(81)$ & $\begin{array}{l}\text { IVB, } 1.25 \mathrm{mg} \text { at baseline } \\
\text { and } 6 \text { weeks }(n=42)\end{array}$ & $\begin{array}{l}\text { Sham injection, at } \\
\text { baseline and } 6 \text { weeks } \\
(n=39)\end{array}$ & $\begin{array}{l}\text { Foveal haemorrhage } \\
\text { Foveal ischemia } \\
\text { Outcomes at } 12 \text { weeks }\end{array}$ & $\begin{array}{l}\text { Methods of IVB } \\
\text { preparation not reported }\end{array}$ \\
\hline \multicolumn{7}{|c|}{ Studies including patients with other ophthalmic conditions ( $n=3$ studies) } \\
\hline $\begin{array}{l}\text { Gharbiya et al, }{ }^{18} \\
\text { Italy }\end{array}$ & $\begin{array}{l}\text { Pathologic myopiat } \\
\text { BCVA, } \geq 26 \text { ETDRS } \\
\text { letters } \\
\text { Mean age, } 59.5 \text { years }\end{array}$ & $32(32)$ & $\begin{array}{l}\text { IVB, } 1.25 \mathrm{mg} \text { at baseline, } \\
\text { then given as needed } \\
\text { ( } \mathrm{n}=16 \text { eyes) }\end{array}$ & $\begin{array}{l}\text { IVR, } 0.5 \mathrm{mg} \text { at } \\
\text { baseline, then given } \\
\text { as needed }(n=16 \\
\text { eyes) }\end{array}$ & $\begin{array}{l}\text { Systemic adverse events } \\
\text { Endophthalmitis Retinal } \\
\text { detachment } \\
\text { Vitreous haemorrhage } \\
\text { Hypertension } \\
\text { IOP } \\
\text { Outcomes at } 6 \text { months }\end{array}$ & $\begin{array}{l}\text { Methods of IVB } \\
\text { preparation not reported }\end{array}$ \\
\hline
\end{tabular}

Study identifier, Description of study study location
Number of

patients

(eyes)

\section{Interventions (treatment Comparators} schedule and numbers treated) 


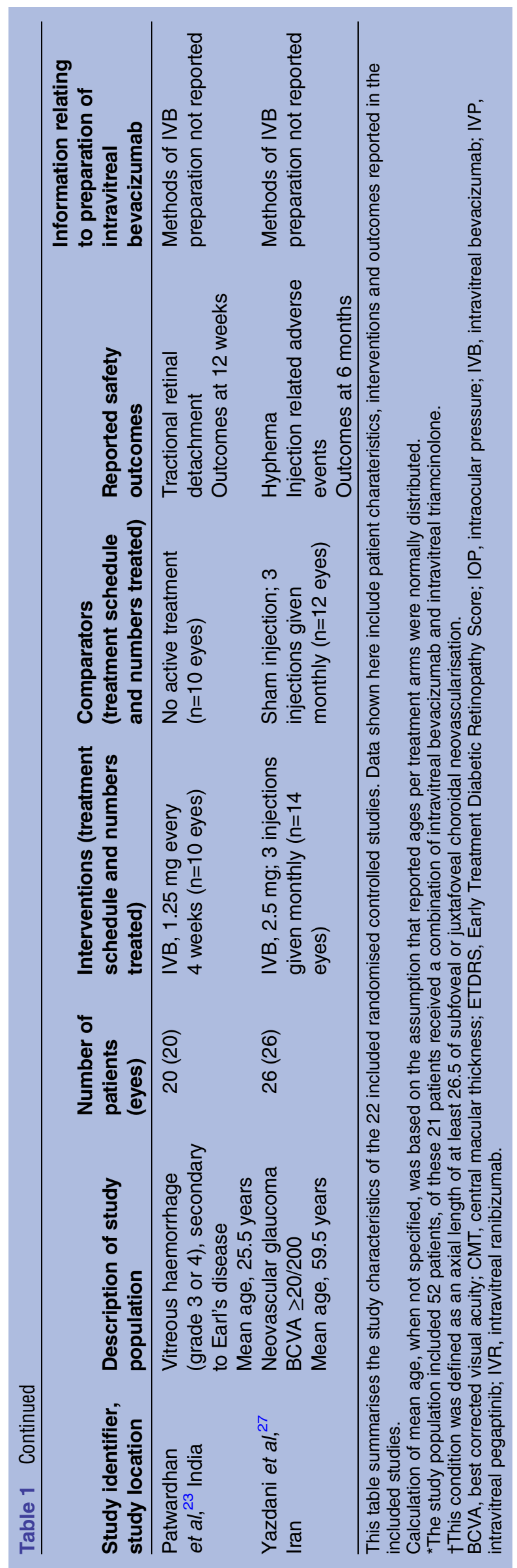

$0.02 \% \quad(\mathrm{n}=3 / 12585$ injections $),{ }^{47} 0.2 \% \quad(\mathrm{n}=1 / 625){ }^{62}$ and $0.8 \%(\mathrm{n}=1 / 112) .{ }^{42} \mathrm{~A}$ higher rate of $0.9 \%(\mathrm{n}=1 / 109)$ was reported in a study with IVB supplied by a compounding pharmacy. ${ }^{48}$ Positive cultures of microorganisms were reported in a study from Fong $e t a l,{ }^{48}$ and another study from Wu et al..$^{94}$

\section{DISCUSSION}

Eighty-nine studies were included in this systematic review of AEs, 22 of which were RCTs. Trials compared IVB with a number of different therapies and eye conditions, though most were in AMD, DMO and RVO. Most ocular and systemic safety measures had zero events in treatment groups or were not significantly different between groups. The quality of reporting of studies made it impossible to evaluate the impact of both known and unknown confounding factors (eg, the use of prophylactic antibiotic eye drops) on the incidence of AEs.

The most robust data for safety are from the CATT ${ }^{16}$ and IVAN $^{19}$ trials which were large trials that reported longer term data. The results of these trials when meta-analysed revealed a statistically significantly higher rate of 1 or more serious systematic AE (RR 1.27; 95\% CI 1.09 to 1.47 ) in the IVB group. In this analysis, the IVAN study ${ }^{19}$ alone did not show a statistically significant difference while event rates were higher in the CATT. The recently published 2-year results of the IVAN study, which was not included in this review, has reported relatively worse safety outcomes for patients on discontinuous treatment compared to continuous treatment. ${ }^{97}$ In addition, there were no observed differences in mortality, frequency of thrombotic events or hospitalisation due to cardiac failure between groups of patients treated with IVB or IVR. Reported pooled analysis of the 2 years results of the CATT and IVAN studies tends to demonstrate that IVB and IVR are comparable in terms of safety. It is also important to note that AEs were more common in those patients who received discontinuous rather than patients on continuous treatment, that is, those with lower exposure to the drug experienced higher $\mathrm{AE}$ rates. An explanation for this observation is the possible role of immunological processes in drug interactions. ${ }^{97}$ It is also important to note that the CATT study demonstrated some imbalances at baseline between randomised patients which may need further exploration. More patients randomised to IVB had had a previous TIA compared to those in the IVR arms. Similarly, more IVB patients had a history of MI. ${ }^{16}$ Despite these caveats, these trial designs offer the most robust assessment of AEs to date.

Overall, the evidence on IVB safety from observational studies was uncertain. This has previously been reported elsewhere. ${ }^{45}$ Included studies were frequently associated with methodological weaknesses that limited the validity of the reported findings. The majority of studies were retrospective in design with small study samples or 
Table 2 Summary of included observational studies

\begin{tabular}{|c|c|c|c|c|c|c|c|c|c|c|}
\hline Author (year) & Study type & $\begin{array}{l}\text { Condition } \\
\text { (patients' mean } \\
\text { age in years) }\end{array}$ & $\begin{array}{l}\text { Number of } \\
\text { patients } \\
\text { (number of } \\
\text { eyes) }\end{array}$ & $\begin{array}{l}\text { Baseline } \\
\text { comparability (yes/ } \\
\text { no/unknown/not } \\
\text { applicable) }\end{array}$ & $\begin{array}{l}\text { Dosage }(\mathrm{mg}) \\
\text { including frequency } \\
\text { of dosing }\end{array}$ & $\begin{array}{l}\text { Number of } \\
\text { injections/ } \\
\text { patients } \\
\text { (mean) }\end{array}$ & Follow-up & $\begin{array}{l}\text { Information } \\
\text { on preparation } \\
\text { of } \\
\text { bevacizumab }\end{array}$ & Funding & Notes \\
\hline $\begin{array}{l}\text { Abraham-Marin } \\
(2007)^{33}\end{array}$ & $\begin{array}{l}\text { Case series, } \\
\text { (prospective) }\end{array}$ & $\begin{array}{l}\text { CNV due to AMD } \\
\text { (76) }\end{array}$ & 39 (39) & NA & $2.5 \mathrm{mg}$ & 1 & 4 weeks & NR & NR & \\
\hline Arevalo $(2010)^{34}$ & $\begin{array}{l}\text { Case series, } \\
\text { (retrospective) }\end{array}$ & CNV due to AMD & $180(207)$ & NA & $\begin{array}{l}1.25 \mathrm{mg}(59.9 \%) \\
2.5 \mathrm{mg}(40.1 \%) \\
\text { Frequency of dosing at } \\
\text { discretion of treating } \\
\text { physician }\end{array}$ & 5.1 (per eye) & $\begin{array}{l}1,3,6,12 \\
\text { and } \\
24 \text { months } \\
\text { after the } \\
\text { initial } \\
\text { injection }\end{array}$ & NR & $\begin{array}{l}\text { Arevalo-Coutinho } \\
\text { Foundation for } \\
\text { Research in } \\
\text { Ophthalmology, } \\
\text { Venezuela }\end{array}$ & \\
\hline Artunay $(2009)^{35}$ & $\begin{array}{l}\text { Case series, } \\
\text { (retrospective) }\end{array}$ & Various* (NR) & NR (1822) & NA & $\begin{array}{l}1.25 \mathrm{mg} \text { once or } \\
\text { repeated }\end{array}$ & NR & $\begin{array}{l}1-7 \text { days, } \\
4 \text { weeks, } \\
8 \text { weeks }\end{array}$ & NR & NR & \\
\hline Azad $(2008)^{36}$ & $\begin{array}{l}\text { Non-randomised trial } \\
\text { (prospective) }\end{array}$ & $\begin{array}{l}\text { subfoveal CNV } \\
\text { due to AMD (63) }\end{array}$ & $40(40)$ & NA & $1.25 \mathrm{mg}$ & 2.4 & 6 months & NR & NR & \\
\hline Baba $(2010)^{37}$ & $\begin{array}{l}\text { Case series } \\
\text { (retrospective) }\end{array}$ & Myopic CNV & $40(40)$ & Yes & $1.25 \mathrm{mg}$ & 1.3 to 1.5 & 24 months & NR & NR & $\begin{array}{l}\text { Treatment groups: } \\
\text { PDT }(n=16) ; \text { PDT } \\
\text { and IVB }(n=12) ; \\
\text { IVB only }(n=12)\end{array}$ \\
\hline Bakri $(2009)^{38}$ & $\begin{array}{l}\text { Case series, } \\
\text { (retrospective) }\end{array}$ & Varioust (NR) & $35(70)$ & NA & $1.25 \mathrm{mg}$ & 5.9 & 39 days & NR & $\begin{array}{l}\text { The Research To } \\
\text { Prevent Blindness, } \\
\text { New York }\end{array}$ & \\
\hline $\begin{array}{l}\text { Bashshur } \\
(2009)^{28}\end{array}$ & $\begin{array}{l}\text { Nonrandomised trial, } \\
\text { open-label, } \\
\text { prospective } \\
\text { (extension study) }\end{array}$ & $\begin{array}{l}\text { CNV due to AMD } \\
(72.2)\end{array}$ & $51(51)$ & NA & $2.5 \mathrm{mg}$ & $\begin{array}{l}2.5 \text { ( } 3.4 \\
\text { during first } \\
12 \text { months, } \\
\text { decreased to } \\
1.5 \text { during } \\
\text { second year) }\end{array}$ & 24 months & $\begin{array}{l}\text { local } \\
\text { dispensing } \\
\text { service }\end{array}$ & $\begin{array}{l}\text { American University of } \\
\text { Beirut Medical Center }\end{array}$ & \\
\hline Carneiro $(2010)^{39}$ & Cohort, (prospective) & $\begin{array}{l}\text { Subfoveal or } \\
\text { juxtafoveal CNV } \\
\text { secondary to } \\
\text { AMD (76.9) }\end{array}$ & (80) & NR & $1.25 \mathrm{mg}$ & 4 & $\begin{array}{l}6 \text { months, } \\
12 \text { months }\end{array}$ & NR & $\begin{array}{l}\text { Sociedade Portuguesa } \\
\text { de Oftalmologia, } \\
\text { Hospitalde Sao Joao, }\end{array}$ & \\
\hline Carneiro $(2011)^{40}$ & $\begin{array}{l}\text { Cohort } \\
\text { (retrospective):IVB } \\
\text { vs IVR }\end{array}$ & AMD (77.8) & 97 (IVB group) & Yes (IVB:IVR) & $1.25 \mathrm{mg}$; & 7.8 & 2.3 years & NR & $\begin{array}{l}\text { Sociedade Portuguesa } \\
\text { de Oftalmologia, } \\
\text { Hospitalde Sao Joao, } \\
\text { Swiss National } \\
\text { Foundation and Walter } \\
\text { and Gertrud } \\
\text { Sienenthaler } \\
\text { Foundation }\end{array}$ & $\begin{array}{l}\text { Increased rate of } \\
\text { ATEs in IVB group } \\
\text { compared to IVT } \\
\text { (secondary } \\
\text { analyses }\end{array}$ \\
\hline Chen $(2010)^{41}$ & $\begin{array}{l}\text { Non-randomised } \\
\text { cohort (retrospective) }\end{array}$ & $\begin{array}{l}\text { MO due to } \\
\text { BRVO (60.7) }\end{array}$ & $24(25)$ & $\begin{array}{l}\text { Yes (IVB:IVT:control; } \\
\mathrm{n}=83 \text { ) }\end{array}$ & $\begin{array}{l}2.5 \mathrm{mg} \text { single injection } \\
\text { then as needed }\end{array}$ & NR & $\begin{array}{l}10 \text { months } \\
\text { (mean) }\end{array}$ & NR & NR & $\begin{array}{l}\text { Patients received } \\
\text { IOP-lowering } \\
\text { treatment during } \\
\text { follow-up period if } \\
\mathrm{IOP} \geq 21 \mathrm{~mm} \mathrm{Hg} \text {. } \\
\text { Anterior } \\
\text { paracentesis was } \\
\text { performed before } \\
\text { IVB to reduce } \\
\text { ocular pressure. }\end{array}$ \\
\hline
\end{tabular}




\begin{tabular}{|c|c|c|c|c|c|c|c|c|c|c|}
\hline Author (year) & Study type & $\begin{array}{l}\text { Condition } \\
\text { (patients' mean } \\
\text { age in years) }\end{array}$ & $\begin{array}{l}\text { Number of } \\
\text { patients } \\
\text { (number of } \\
\text { eyes) }\end{array}$ & $\begin{array}{l}\text { Baseline } \\
\text { comparability (yes/ } \\
\text { no/unknown/not } \\
\text { applicable) }\end{array}$ & $\begin{array}{l}\text { Dosage }(\mathrm{mg}) \\
\text { including frequency } \\
\text { of dosing }\end{array}$ & $\begin{array}{l}\text { Number of } \\
\text { injections/ } \\
\text { patients } \\
\text { (mean) }\end{array}$ & Follow-up & $\begin{array}{l}\text { Information } \\
\text { on preparation } \\
\text { of } \\
\text { bevacizumab }\end{array}$ & Funding & Notes \\
\hline & & & & & & & & & & $\begin{array}{l}\text { Author's } \\
\text { conclusion: } \\
\text { IVB better than IVT }\end{array}$ \\
\hline Cleary $(2008)^{42}$ & $\begin{array}{l}\text { Case series } \\
\text { (retrospective) }\end{array}$ & $\begin{array}{l}\text { Neovascular } \\
\text { AMD (75) }\end{array}$ & 111 (112) & NA & $\begin{array}{l}1.25 \mathrm{mg} \text {, once then as } \\
\text { needed }\end{array}$ & NR & $\begin{array}{l}4.9 \text { (range } \\
1-12 \text { ) }\end{array}$ & $\begin{array}{l}\text { Local } \\
\text { dispensing } \\
\text { service }\end{array}$ & None & \\
\hline Costa $(2006)^{43}$ & $\begin{array}{l}\text { Non-randomised } \\
\text { dose escalation study } \\
\text { (prospective) }\end{array}$ & $\begin{array}{l}\text { CNV caused by } \\
\text { AMD (74.6) }\end{array}$ & $45(45)$ & $\begin{array}{l}\text { Yes } \\
(1.0 \mathrm{mg}: 1.5 \mathrm{mg}: 2.0 \mathrm{mg})\end{array}$ & $\begin{array}{l}1.0 \mathrm{mg}, 1.5 \mathrm{mg} \text { and } \\
2.0 \mathrm{mg}\end{array}$ & NR & 3 & $\begin{array}{l}\text { Local } \\
\text { dispensing } \\
\text { service }\end{array}$ & $\begin{array}{l}\text { Public funding } \\
\text { (Foundation for } \\
\text { Research Support of } \\
\text { the State of São } \\
\text { Paulo) }\end{array}$ & $\begin{array}{l}\text { Reported as a } \\
\text { dose escalation } \\
\text { study but difficult to } \\
\text { tell how many } \\
\text { doses each } \\
\text { participant was } \\
\text { given and how far } \\
\text { apart }\end{array}$ \\
\hline $\begin{array}{l}\text { Costagliola } \\
(2009)^{44}\end{array}$ & $\begin{array}{l}\text { Case series } \\
\text { (retrospective) }\end{array}$ & $\begin{array}{l}\text { CNV (subfoveal) } \\
\text { due to AMD } \\
\text { (73.2) }\end{array}$ & $68(68)$ & NA & $\begin{array}{l}1.25 \text {; then monthly as } \\
\text { per needed }\end{array}$ & $\begin{array}{l}3.87 \text { (first } \\
6 \text { months); } \\
1.09 \text { (for } \\
\text { remaining } \\
6 \text { months) }\end{array}$ & 12 & $\begin{array}{l}\text { Local } \\
\text { dispensing } \\
\text { service }\end{array}$ & NR & $\begin{array}{l}\text { Exclusion criteria } \\
\text { included previous } \\
\text { history of } \\
\text { thromboembolic } \\
\text { events; } \\
\text { uncontrolled } \\
\text { hypertension, BP } \\
>150 / 90 \mathrm{~mm} \mathrm{Hg} \text {. } \\
\text { Topical antibiotics } \\
\text { prescribed for } \\
3 \text { days, after } \\
\text { injection }\end{array}$ \\
\hline Curtis $(2010)^{45}$ & Cohort (retrospective) & $\begin{array}{l}\text { AMD (median, } \\
81.0 \text { ) }\end{array}$ & $\begin{array}{l}27962 \text { (IVB } \\
\text { only; } \\
n=146942)\end{array}$ & $\begin{array}{l}\text { Yes (IVB:PDT: IVP: } \\
\text { IVR) }\end{array}$ & NR & NR & 12 months & NR & $\begin{array}{l}\text { Research agreement } \\
\text { between OSI Eyetech } \\
\text { and Duke University }\end{array}$ & $\begin{array}{l}\text { Patient data were } \\
\text { censored when at } \\
\text { the time when a } \\
\text { treatment which } \\
\text { was different from } \\
\text { initially assigned } \\
\text { intervention was } \\
\text { received. Between } \\
\text { July and } \\
\text { December } 2006 \text {, } \\
\text { study population } \\
\text { was limited to } \\
\text { treatment-nä̈ve } \\
\text { patients who } \\
\text { received } \\
\text { bevacizumab or } \\
\text { ranibizumab }\end{array}$ \\
\hline $\begin{array}{l}\text { Falkenstein } \\
(2007)^{46}\end{array}$ & $\begin{array}{l}\text { Case series } \\
\text { (prospective) }\end{array}$ & AMD (79.4) & 70 (NR) & NA & $\begin{array}{l}1.25 \mathrm{mg} \text { assumed } \\
(0.05 \mathrm{~mL})\end{array}$ & $\begin{array}{l}1.74 \\
\text { (calculated } \\
\text { from } 122 \\
\text { injections for } \\
70 \text { patients) }\end{array}$ & $\begin{array}{l}3,10 \text { and } 15 \\
\text { minutes }\end{array}$ & NR & NR & \\
\hline
\end{tabular}




\begin{tabular}{|c|c|c|c|c|c|c|c|c|c|c|}
\hline Author (year) & Study type & $\begin{array}{l}\text { Condition } \\
\text { (patients' mean } \\
\text { age in years) }\end{array}$ & $\begin{array}{l}\text { Number of } \\
\text { patients } \\
\text { (number of } \\
\text { eyes) }\end{array}$ & $\begin{array}{l}\text { Baseline } \\
\text { comparability (yes/ } \\
\text { no/unknown/not } \\
\text { applicable) }\end{array}$ & $\begin{array}{l}\text { Dosage }(\mathrm{mg}) \\
\text { including frequency } \\
\text { of dosing }\end{array}$ & $\begin{array}{l}\text { Number of } \\
\text { injections/ } \\
\text { patients } \\
\text { (mean) }\end{array}$ & Follow-up & $\begin{array}{l}\text { Information } \\
\text { on preparation } \\
\text { of } \\
\text { bevacizumab }\end{array}$ & Funding & Notes \\
\hline Fintak $(2008)^{47}$ & cohort (retrospective) & Various (NR) & $\begin{array}{l}12585 \text { (IVB } \\
\text { injections) }\end{array}$ & NR & $1.25 \mathrm{mg}$ & NR & 5 days & $\begin{array}{l}\text { Local } \\
\text { dispensing } \\
\text { service }\end{array}$ & NR & $\begin{array}{l}\text { Number of } \\
\text { injections not } \\
\text { reported }\end{array}$ \\
\hline Fong $(2008)^{48}$ & $\begin{array}{l}\text { Case series } \\
\text { (retrospective) }\end{array}$ & AMD (82) & 109 (109) & NA & $\begin{array}{l}1.25 \mathrm{mg} \text {, three } \\
\text { consecutive monthly } \\
\text { injections then as } \\
\text { needed }\end{array}$ & NR & $\begin{array}{l}9.4 \text { months } \\
\text { (range 6-12) }\end{array}$ & $\begin{array}{l}\text { Compounding } \\
\text { pharmacy }\end{array}$ & NR & \\
\hline Frenkel $2010^{49}$ & Cohort (retrospective) & AMD (80) & $47 \ddagger$ & $\begin{array}{l}\text { Unknown (IVB: } \\
\text { ranibizumab: } \\
\text { pegaptanib) }\end{array}$ & $1.25 \mathrm{mg}$ & 1 & 20 minutes & NR & NR & $\begin{array}{l}\text { First injection only } \\
\text { selected for the } \\
\text { study }\end{array}$ \\
\hline $\begin{array}{l}\text { Fukami }(2011)^{50} \\
\text { (abstract) }\end{array}$ & $\begin{array}{l}\text { Case series } \\
\text { (retrospective) }\end{array}$ & NR (NR) & $12(12)$ & NA & NR & NR & 2 days & NR & NR & \\
\hline $\begin{array}{l}\text { Gamulescu } \\
(2010)^{51}\end{array}$ & $\begin{array}{l}\text { Cohort } \\
\text { (retrospective)§ }\end{array}$ & AMD (77.5) & 30 (NR) & NR & $\begin{array}{l}1.25 \mathrm{mg} \text { every } 4 \text { weeks } \\
3 \text { initial injections }\end{array}$ & NR & $\begin{array}{l}2-4 \text { months } \\
\text { after last } \\
\text { injection }\end{array}$ & NR & NR & \\
\hline Gomi $(2008)^{52}$ & $\begin{array}{l}\text { Case series } \\
\text { (Retrospective) }\end{array}$ & $\begin{array}{l}\text { Polypoidal } \\
\text { choroidal } \\
\text { vasculopathy } \\
(65.4)\end{array}$ & $11(11)$ & NA & $\begin{array}{l}1 \mathrm{mg} \text { f once or as } \\
\text { needed }\end{array}$ & NR & $\begin{array}{l}9.4 \text { months } \\
( \pm 4.4)\end{array}$ & NR & NR & \\
\hline Good $(2011)^{31}$ & 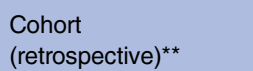 & AMD (76.6) & NR (101)†† & Yes & $1.25 \mathrm{mg}$ & 7.0 & $\begin{array}{l}86.6 \text { days } \\
\text { mean }\end{array}$ & NR & NR & \\
\hline $\begin{array}{l}\text { Goverdhan } \\
(2008)^{53}\end{array}$ & $\begin{array}{l}\text { Case series } \\
\text { (retrospective) }\end{array}$ & $\begin{array}{l}\text { CNV due to AMD } \\
\text { (79.5) }\end{array}$ & $53(53)$ & NA & $\begin{array}{l}1.25 \mathrm{mg} \\
\text { Repeat injections } \\
\text { offered if CNV } \\
\text { persisted or fresh } \\
\text { haemorrhage or } \\
\text { subretinal fluid } \\
\text { observed. }\end{array}$ & 1.36 & $\begin{array}{l}\text { Day } 1 \text { and } \\
\text { after } 2 \text { week } \\
\text { visits then at } \\
4 \text {-week } \\
\text { intervals. } \\
\text { Minimum } \\
6 \text { months } \\
\text { (range } 4 \text { to } \\
12 \text { months) }\end{array}$ & NR & NR & \\
\hline $\begin{array}{l}\text { Gower }(2011)^{54} \\
\text { (abstract) }\end{array}$ & Cohort (retrospective) & $\begin{array}{l}\text { Neovascular } \\
\text { AMD (NR) }\end{array}$ & NR (NR) & NR (IVB:IVR) & NR & NR & NR & NR & NR & $\begin{array}{l}\text { HRs adjusted for } \\
\text { baseline } \\
\text { comorbidities, } \\
\text { demographics and } \\
\text { socio-economic } \\
\text { status }\end{array}$ \\
\hline $\begin{array}{l}\text { Hernandez-Rojas } \\
(2007)^{55}\end{array}$ & $\begin{array}{l}\text { Case series } \\
\text { (prospective) }\end{array}$ & $\begin{array}{l}\text { CNV due to } \\
\text { pathological } \\
\text { myopia (53.9) }\end{array}$ & $\begin{array}{l}13 \text { (13) (at } \\
\text { follow-up-one } \\
\text { patients lost to } \\
\text { follow-up) }\end{array}$ & NA & $\begin{array}{l}2.5 \mathrm{mg} / 0.1 \mathrm{~mL} \text { once or } \\
\text { as needed }\end{array}$ & NR & 3 months & NR & NR & \\
\hline $\begin{array}{l}\text { Higashide } \\
(2012)^{56}\end{array}$ & $\begin{array}{l}\text { Case series } \\
\text { (retrospective) }\end{array}$ & $\begin{array}{l}\text { Neovascular } \\
\text { glaucoma (63.5) }\end{array}$ & $70(84)$ & NA & $1.25 \mathrm{mg}$ & 1.4 & 3 months & NR & NR & \\
\hline Hollands $(2007)^{57}$ & $\begin{array}{l}\text { Case series } \\
\text { (prospective) }\end{array}$ & $\begin{array}{l}\text { Neovascular } \\
\text { AMD }(84.6 \%) \text {; } \\
\text { DMO }(6.7 \%) \text {; } \\
\text { Others- } \\
\text { histoplasmosis } \\
(8.7 \%)(76)\end{array}$ & 104 & NA & $1.25 \mathrm{mg}$ & NR & $30 \mathrm{~min}$ & NR & NR & \\
\hline
\end{tabular}




\begin{tabular}{|c|c|c|c|c|c|c|c|c|c|c|}
\hline Author (year) & Study type & $\begin{array}{l}\text { Condition } \\
\text { (patients' mean } \\
\text { age in years) }\end{array}$ & $\begin{array}{l}\text { Number of } \\
\text { patients } \\
\text { (number of } \\
\text { eyes) }\end{array}$ & $\begin{array}{l}\text { Baseline } \\
\text { comparability (yes/ } \\
\text { no/unknown/not } \\
\text { applicable) }\end{array}$ & $\begin{array}{l}\text { Dosage }(\mathrm{mg}) \\
\text { including frequency } \\
\text { of dosing }\end{array}$ & $\begin{array}{l}\text { Number of } \\
\text { injections/ } \\
\text { patients } \\
\text { (mean) }\end{array}$ & Follow-up & $\begin{array}{l}\text { Information } \\
\text { on preparation } \\
\text { of } \\
\text { bevacizumab }\end{array}$ & Funding & Notes \\
\hline Ikuno $(2009)^{58}$ & $\begin{array}{l}\text { Case series } \\
\text { (retrospective) }\end{array}$ & $\begin{array}{l}\text { CNV due to } \\
\text { myopia (58.4) }\end{array}$ & $63(63)$ & NA & $1 \mathrm{mg}$ & 2.4 & 12 months & NR & $\begin{array}{l}\text { The Ministry of } \\
\text { Education, Culture, } \\
\text { Sports Science and } \\
\text { Technology of Japan; } \\
\text { Health and Labor } \\
\text { Sciences Research of } \\
\text { Japan }\end{array}$ & $\begin{array}{l}\text { Re-injection } \\
\text { considered after 2- } \\
3 \text { months if } \\
\text { fluorescein leakage } \\
\text { in angiograam or } \\
\text { subretinal fluid } \\
\text { persisted }\end{array}$ \\
\hline Inman $(2011)^{59}$ & $\begin{array}{l}\text { Case series } \\
\text { (retrospective) }\end{array}$ & NR & $\begin{array}{l}608 \text { (sample } \\
\text { included } \\
\text { patients that } \\
\text { received IVB, } \\
\text { IVP and IVR) }\end{array}$ & NA & NR & $\begin{array}{l}\text { Unclear } \\
\text { (1841 } \\
\text { injections of } \\
\text { IVB, 428 IVP } \\
\text { and } 2421 \\
\text { IVR) }\end{array}$ & 4.4 years & $\begin{array}{l}\text { Local } \\
\text { dispensing } \\
\text { service }\end{array}$ & NR & $\begin{array}{l}\text { This study reported } \\
\text { incidence of } \\
\text { infectious } \\
\text { endophthalmitis } \\
\text { associated with } 2 \% \\
\text { topical lidocaine } \\
\text { gel anaesthesia. } \\
\text { No information on } \\
\text { conditions being } \\
\text { treated or patient } \\
\text { demographics. }\end{array}$ \\
\hline Jaissle $(2009)^{60}$ & $\begin{array}{l}\text { Case series } \\
\text { (prospective) }\end{array}$ & $\begin{array}{l}\text { MO due to } \\
\text { BRVO (median, } \\
68 \text { ) }\end{array}$ & $23(23)$ & NA & $\begin{array}{l}1.25 \mathrm{mg} \text { (re-injection } \\
\text { considered if macular } \\
\text { oedema persisted in } \\
\text { foveal area and visual } \\
\text { acuity } 20 / 32 \text { or worse) }\end{array}$ & NR & $\begin{array}{l}1 \text { year. } \\
\text { (examined } \\
\text { every } \\
6 \text { weeks) }\end{array}$ & NR & $\begin{array}{l}\text { German } \\
\text { Opthalmological } \\
\text { Society }\end{array}$ & $\begin{array}{l}\text { During the } 1 \text {-year } \\
\text { follow-up, an } \\
\text { average of } 2.4 \\
\text { re-injections } \\
\text { (range, } 0-5 \text { ) were } \\
\text { administered, with } \\
\text { a mean of } 1.6 \\
\text { re-injections within } \\
\text { the first } 6 \text { months } \\
\text { (weeks } 6-24 \text { ) and } \\
\text { a further } 0.8 \\
\text { re-injections over } \\
\text { the latter } 6 \text { months } \\
\text { (weeks } 30-48 \text { ). }\end{array}$ \\
\hline Johnson $(2010)^{61}$ & $\begin{array}{l}\text { Case series } \\
\text { (retrospective) }\end{array}$ & Variousł‡ (76.5) & $173(193)$ & NA & NR & 3.98 & $\begin{array}{l}\text { Median } \\
\text { follow-up; } \\
40 \text { days } \\
\text { (range } 19 \text { to } \\
170 \text { days) }\end{array}$ & NR & $\begin{array}{l}\text { Queen's University, } \\
\text { Canada }\end{array}$ & \\
\hline Jonas $(2007)^{62}$ & $\begin{array}{l}\text { Case series } \\
\text { (retrospective) }\end{array}$ & AMD & $625(684)$ & NA & $1.5 \mathrm{mg}$ & 1.95 & $\geq 4$ weeks & $\begin{array}{l}\text { Local } \\
\text { dispensing } \\
\text { service }\end{array}$ & NR & 534 re-injections \\
\hline Jonas $(2008)^{63}$ & $\begin{array}{l}\text { Case series } \\
\text { (retrospective, } \\
\text { consecutive) }\end{array}$ & Various & $\begin{array}{l}\text { NR (3818 IVB } \\
\text { injections) }\end{array}$ & NA & $1.5 \mathrm{mg}$ & NR & $\geq 3$ months & NR & None & \\
\hline Julian $(2011)^{64}$ & $\begin{array}{l}\text { Case series } \\
\text { (retrospective) }\end{array}$ & $\begin{array}{l}\text { CNV due to } \\
\text { uveitis (median, } \\
41.9 \text { ) }\end{array}$ & $15(15)$ & NA & $\begin{array}{l}1.25 \mathrm{mg} \text { (re-treatment } \\
\text { based on signs of } \\
\text { active } \\
\text { neovascularisation) }\end{array}$ & 4.25 & 17.6 (median) & NR & NR & $\begin{array}{l}\text { In all cases, } \\
\text { optimum control of } \\
\text { intraocular } \\
\text { inflammation was } \\
\text { achieved by the } \\
\text { time IVB was } \\
\text { initiated }\end{array}$ \\
\hline
\end{tabular}




\begin{tabular}{|c|c|c|c|c|c|c|c|c|c|c|}
\hline Author (year) & Study type & $\begin{array}{l}\text { Condition } \\
\text { (patients' mean } \\
\text { age in years) }\end{array}$ & $\begin{array}{l}\text { Number of } \\
\text { patients } \\
\text { (number of } \\
\text { eyes) }\end{array}$ & $\begin{array}{l}\text { Baseline } \\
\text { comparability (yes/ } \\
\text { no/unknown/not } \\
\text { applicable) }\end{array}$ & $\begin{array}{l}\text { Dosage }(\mathrm{mg}) \\
\text { including frequency } \\
\text { of dosing }\end{array}$ & $\begin{array}{l}\text { Number of } \\
\text { injections/ } \\
\text { patients } \\
\text { (mean) }\end{array}$ & Follow-up & $\begin{array}{l}\text { Information } \\
\text { on preparation } \\
\text { of } \\
\text { bevacizumab }\end{array}$ & Funding & Notes \\
\hline $\operatorname{Kim}(2009)^{32}$ & $\begin{array}{l}\text { Before-after study of } \\
\text { IVB group and } \\
\text { triamcinolone } \\
\text { acetonide group } \\
\text { (retrospective) }\end{array}$ & $\begin{array}{l}\text { MO due to } \\
\text { BRVO (56.9) }\end{array}$ & $\begin{array}{l}50(50)(22 \\
\text { received IVB } \\
\text { and } 28 \\
\text { received } \\
\text { triamcinolone } \\
\text { acetonide) }\end{array}$ & NA & $1.25 \mathrm{mg}$ single dose & NR & 24 weeks & NR & NR & NR \\
\hline $\operatorname{Kim}(2011)^{65}$ & $\begin{array}{l}\text { Case series } \\
\text { (retrospective) }\end{array}$ & DMO & $48(65)$ & Yes & $1.25 \mathrm{mg}$ & $\mathrm{NR}$ & $\geq 12$ months & NR & $\begin{array}{l}\text { Grant from Kyung Hee } \\
\text { University }\end{array}$ & \\
\hline $\operatorname{Kim}(2011)^{29}$ & $\begin{array}{l}\text { Non-randomised } \\
\text { controlled study } \\
\text { (prospective, } \\
\text { consecutive) }\end{array}$ & $\begin{array}{l}\text { AMD, RVO, } \\
\text { DMO (64.8) }\end{array}$ & $60(60)$ & Yes & $1.25 \mathrm{mg}$ & 1 & NR & NR & NR & \\
\hline Kiss $(2006)^{66}$ & $\begin{array}{l}\text { case-control } \\
\text { (retrospective)§§ }\end{array}$ & AMD (NR) & 61 & Yes & $1 \mathrm{mg}$ & 1 & 7 days & $\begin{array}{l}\text { Local } \\
\text { dispensing } \\
\text { service }\end{array}$ & NR & \\
\hline Krebs $(2009)^{67}$ & $\begin{array}{l}\text { Case series } \\
\text { (prospective) }\end{array}$ & AMD (NR) & $44(44)$ & Unknown & $\begin{array}{l}1.25 \mathrm{mg} 3 \text { monthly } \\
\text { injections based on } \\
\text { OCT and FA findings }\end{array}$ & 2.6 & $\begin{array}{l}1 \text { week, } \\
1 \text { month and } \\
3 \text { months }\end{array}$ & NR & L. Boltzmann Institute & \\
\hline $\begin{array}{l}\text { Kriechbaum } \\
(2008)^{68}\end{array}$ & $\begin{array}{l}\text { Case series } \\
\text { (prospective) }\end{array}$ & $\begin{array}{l}\text { MO due to } \\
\text { BRVO or CRVO } \\
(66)\end{array}$ & $28(29)$ & Unknown & $\begin{array}{l}1 \mathrm{mg} \text { at } 4 \text {-week } \\
\text { intervals } 3 \text { intravitreal } \\
\text { injections }\end{array}$ & 5.3 & $\begin{array}{l}1,7 \text { and } \\
28 \text { months }\end{array}$ & $\begin{array}{l}\text { Local } \\
\text { dispensing } \\
\text { service }\end{array}$ & NR & \\
\hline Krishnan (2009) $)^{69}$ & $\begin{array}{l}\text { Case control } \\
\text { (retrospective) १ๆ }\end{array}$ & $\begin{array}{l}\text { CNV due to AMD } \\
(80.5)\end{array}$ & 14 & No & $1.25 \mathrm{mg}$ & NR & $\begin{array}{l}2 \text { and } \\
4 \text { weeks }\end{array}$ & NR & NR & \\
\hline Kumar $(2012)^{70}$ & $\begin{array}{l}\text { Case series } \\
\text { (retrospective) }\end{array}$ & $\begin{array}{l}\text { Eales' disease } \\
\text { (median, 33) }\end{array}$ & $14(14)$ & Unknown & $1.25 \mathrm{mg}$ & 1 & 3 months & NR & NR & \\
\hline Lazic $(2007)^{71}$ & $\begin{array}{l}\text { Case series } \\
\text { (prospective) }\end{array}$ & $\begin{array}{l}\text { CNV secondary } \\
\text { to AMD }\end{array}$ & $102(102)$ & NA & $\begin{array}{l}1.25 \mathrm{mg} \text {, once then as } \\
\text { needed }\end{array}$ & NR & $\geq 1.5$ months & NR & None & $\begin{array}{l}\text { Follow-up was } \\
6 \text {-weekly and } \\
\text { ongoing }\end{array}$ \\
\hline Lima $(2009)^{72}$ & $\begin{array}{l}\text { Retrospective cohort } \\
\text { study }\end{array}$ & $\begin{array}{l}\text { Various, mostly } \\
\text { AMD }\end{array}$ & $\begin{array}{c}326 \text { (IVB } \\
\text { injections) }\end{array}$ & NR & NR & NR & NR & NR & $\begin{array}{l}\text { Macula Foundation } \\
\text { Inc. }\end{array}$ & $\begin{array}{l}\text { Same-day bilateral } \\
\text { injections }\end{array}$ \\
\hline $\begin{array}{l}\text { Lommatzsch } \\
(2009)^{73}\end{array}$ & $\begin{array}{l}\text { Case series } \\
\text { (retrospective) }\end{array}$ & AMD (77.7) & 86 & NR & $\begin{array}{l}1.25 \mathrm{mg} \text { at } 6 \text { week } \\
\text { intervals }\end{array}$ & NR & 42.4 weeks & NR & NR & \\
\hline Lorenz $(2010)^{74}$ & $\begin{array}{l}\text { Case series } \\
\text { (retrospective) }\end{array}$ & Various ${ }^{\star \star \star}$ & $144(145)$ & Yes & $1.25 \mathrm{mg}$ & 1.63 & 14 & $\begin{array}{l}\text { local } \\
\text { dispensing } \\
\text { service }\end{array}$ & None & \\
\hline Mason $(2008)^{75}$ & $\begin{array}{l}\text { Case series } \\
\text { (retrospective) }\end{array}$ & Varioustt† & NR & NR & $1.25 \mathrm{mg}$ & NR & NR & NR & $\begin{array}{l}\text { University research } \\
\text { grant, New York. }\end{array}$ & \\
\hline $\begin{array}{l}\text { Manayath } \\
(2009)^{76}\end{array}$ & $\begin{array}{l}\text { Case series } \\
\text { (prospective) }\end{array}$ & $\begin{array}{l}\text { CMO due to } \\
\text { CRVO } \\
15 \text { (64) }\end{array}$ & 15 & No & $1.25 \mathrm{mg}$ & 2.2 & $6-18$ months & NR & NR & \\
\hline Rasier $(2009)^{77}$ & $\begin{array}{l}\text { Quasi-experimental } \\
\ddagger \neq \ddagger\end{array}$ & $\operatorname{AMD}(67.2)$ & 82 & Unknown & $1.25 \mathrm{mg}$ & 1 & 6 weeks & NR & NR & \\
\hline Russo $(2009)^{78}$ & $\begin{array}{l}\text { Non-randomised } \\
\text { controlled trial }\end{array}$ & $\begin{array}{l}\text { MO due to } \\
\text { BRVO }\end{array}$ & $15(15)$ & Yes (IVB:LGP) & $\begin{array}{l}1.25 \mathrm{mg} \text {, once or } \\
\text { repeated as necessary }\end{array}$ & NR & 12 months & NR & NR & $\begin{array}{l}\text { No. of eyes/ } \\
\text { patients refers to } \\
\text { IVB group }\end{array}$ \\
\hline Saeed $(2011)^{79}$ & Cohort (prospective) & $\begin{array}{l}\text { Retinal vascular } \\
\text { occlusions and } \\
\text { other causes of } \\
\text { CMO (68.6) }\end{array}$ & 18 & NA & $1.25 \mathrm{mg}$ & NR & NR & NR & NR & $\begin{array}{l}\text { Authors reported } \\
\text { that nti-VEGF } \\
\text { related reflux was } \\
\text { not associated with } \\
\text { a sub-therapeutic } \\
\text { effect }\end{array}$ \\
\hline
\end{tabular}




\begin{tabular}{|c|c|c|c|c|c|c|c|c|c|c|}
\hline Author (year) & Study type & $\begin{array}{l}\text { Condition } \\
\text { (patients' mean } \\
\text { age in years) }\end{array}$ & $\begin{array}{l}\text { Number of } \\
\text { patients } \\
\text { (number of } \\
\text { eyes) }\end{array}$ & $\begin{array}{l}\text { Baseline } \\
\text { comparability (yes/ } \\
\text { no/unknown/not } \\
\text { applicable) }\end{array}$ & $\begin{array}{l}\text { Dosage }(\mathrm{mg}) \\
\text { including frequency } \\
\text { of dosing }\end{array}$ & $\begin{array}{l}\text { Number of } \\
\text { injections/ } \\
\text { patients } \\
\text { (mean) }\end{array}$ & Follow-up & $\begin{array}{l}\text { Information } \\
\text { on preparation } \\
\text { of } \\
\text { bevacizumab }\end{array}$ & Funding & Notes \\
\hline Shah $(2011)^{80}$ & Cohort (retrospective) & Various & $\begin{array}{l}10958 \text { (IVB } \\
\text { injections) }\end{array}$ & NR & NR & NR & 6 days & NR & NR & \\
\hline Sharma $(2012)^{81}$ & Cohort (retrospective) & $\begin{array}{l}\text { AMD, DMO RVO } \\
\text { (IVB group,76.9) }\end{array}$ & $\begin{array}{l}173 \text { (693 IVB } \\
\text { injections) }\end{array}$ & $\begin{array}{l}\text { No difference in age } \\
\text { and VA (IVB:IVR) }\end{array}$ & $1 \mathrm{mg}$ & unclear & NR & $\begin{array}{l}\text { Local } \\
\text { dispensing } \\
\text { service }\end{array}$ & $\begin{array}{l}\text { Part-funded by } \\
\text { Novartis (and } \\
\text { part-funded by } \\
\text { Canadian Institutes for } \\
\text { Health Research) }\end{array}$ & $\begin{array}{l}\text { IVR patients were } \\
\text { on average } \\
1.8 \text { years } \\
\text { older than IVB } \\
\text { patients (78.7 vs } \\
76.9, p \\
0.01 \text { ) and had } \\
\text { slightly worse } \\
\text { baseline vision (6/ } \\
76 \\
\text { vs } 6 / 64, \mathrm{p} 0.013 \text { ). } \\
195 \text { out of the } 351 \\
\text { patients that } \\
\text { received IVR, had } \\
\text { been treated } \\
\text { previously with IVB } \\
\text { (mean, } 4.3 \\
\text { injections per } \\
\text { patient). Prior } \\
\text { treatment in IVB } \\
\text { group unclear }\end{array}$ \\
\hline $\begin{array}{l}\text { Shienbaum } \\
(2012)^{82}\end{array}$ & $\begin{array}{l}\text { Case series } \\
\text { (retrospective) }\end{array}$ & AMD & $73(74)$ & Yes (IVB:IVR) & $\begin{array}{l}\text { NR (Monthly treatment } \\
\text { until no intraretinal or } \\
\text { subretinal fluid on } \\
\text { optical coherence } \\
\text { tomography. Treatment } \\
\text { intervals determined by } \\
\text { signs of exudation }\end{array}$ & NR & 1.41 years & NR & None reported & \\
\hline Shima $(2008)^{83}$ & $\begin{array}{l}\text { Case series } \\
\text { (retrospective) }\end{array}$ & Various§§§ & $\begin{array}{l}707 \text { (1300 } \\
\text { injections) }\end{array}$ & NR & $\begin{array}{l}1 \mathrm{mg} \\
\text { Once or repeated } \\
\text { injections }\end{array}$ & NR & $\geq 2$ months & NR & $\begin{array}{l}\text { Health } \\
\text { Sciences Research } \\
\text { Grant, Ministry of } \\
\text { Health, Labour and } \\
\text { Welfare, Japan }\end{array}$ & \\
\hline Shimada $(2011)^{84}$ & $\begin{array}{l}\text { Case series } \\
\text { (retrospective) }\end{array}$ & $\begin{array}{l}\text { Myopic CNV } \\
(58.4)\end{array}$ & $74(74)$ & NA & $\begin{array}{l}1.25 \mathrm{mg} \\
\text { At baseline, week } 1 \text {, } \\
\text { then monthly } \\
\text { (unspecified length of } \\
\text { time) }\end{array}$ & NR & $\begin{array}{l}12 \text { months } \\
\text { (SD-4.3) }\end{array}$ & NR & $\begin{array}{l}\text { Grants } 19390441 \text { and } \\
19659445 \text { from the } \\
\text { Japan Society for the } \\
\text { Promotion of Science, } \\
\text { Tokyo, Japan }\end{array}$ & \\
\hline Sivkova $(2010)^{85}$ & $\begin{array}{l}\text { case series } \\
\text { (prospective) }\end{array}$ & $\begin{array}{l}\text { CME due to DR, } \\
\text { BRVO and } \\
\text { CRVO (DR } \\
\text { patients } 59.7 ; \\
\text { RVO patients, } \\
68 \text { ) }\end{array}$ & $96(107)$ & Unclear (DR:RVO) & $\begin{array}{l}1.25 \mathrm{mg} 3 \text { consecutive } \\
\text { injections at } 1 \text {-monthly } \\
\text { intervals }\end{array}$ & NR & 4 months & NR & NR & $\begin{array}{l}\text { No significant } \\
\text { difference in } \\
\text { adverse events } \\
\text { between groups }\end{array}$ \\
\hline Sohn $(2011)^{86}$ & $\begin{array}{l}\text { Case control } \\
\text { (prospective) ๆ११ๆ }\end{array}$ & DMO (54.5) & 11 & NA & $1.25 \mathrm{mg}$ & NR & 1.3 months & NR & $\begin{array}{l}\text { GachonUniveristy, } \\
\text { Incheon Korea }\end{array}$ & \\
\hline
\end{tabular}




\begin{tabular}{|c|c|c|c|c|c|c|c|c|c|c|}
\hline Author (year) & Study type & $\begin{array}{l}\text { Condition } \\
\text { (patients' mean } \\
\text { age in years) }\end{array}$ & $\begin{array}{l}\text { Number of } \\
\text { patients } \\
\text { (number of } \\
\text { eyes) }\end{array}$ & $\begin{array}{l}\text { Baseline } \\
\text { comparability (yes/ } \\
\text { no/unknown/not } \\
\text { applicable) }\end{array}$ & $\begin{array}{l}\text { Dosage }(\mathrm{mg}) \\
\text { including frequency } \\
\text { of dosing }\end{array}$ & $\begin{array}{l}\text { Number of } \\
\text { injections/ } \\
\text { patients } \\
\text { (mean) }\end{array}$ & Follow-up & $\begin{array}{l}\text { Information } \\
\text { on preparation } \\
\text { of } \\
\text { bevacizumab }\end{array}$ & Funding & Notes \\
\hline Song $(2011)^{87}$ & 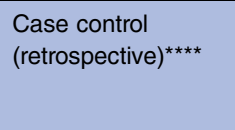 & $\mathrm{DMO}(57.1)$ & $35(58)$ & Yes (IVB:IVT) & $1.25 \mathrm{mg}$ & NR & 8 weeks & NR & $\begin{array}{l}\text { Institute for Medicine } \\
\text { research grant of } \\
\text { Kosin University } \\
\text { College of Medicine }\end{array}$ & \\
\hline Sonmez $(2011)^{88}$ & $\begin{array}{l}\text { Case series } \\
\text { (prospective) }\end{array}$ & $\begin{array}{l}\text { Subfoveal CMO } \\
\text { due to AMD } \\
\text { (69.4) }\end{array}$ & $24(24)$ & NA & $\begin{array}{l}1.25 \mathrm{mg} \text { weeks } 0,6 \\
\text { and } 12 \text {, then every } \\
12 \text { weeks until week } 48\end{array}$ & 5 & NR & NR & NR & $\begin{array}{l}\text { Of } 27 \text { patients, } 3 \\
\text { were lost to } \\
\text { follow-up/protocol } \\
\text { violation) }\end{array}$ \\
\hline Spandau $(2006)^{89}$ & $\begin{array}{l}\text { Case series } \\
\text { (retrospective, } \\
\text { consecutive) }\end{array}$ & AMD & 63 & NA & $1.5 \mathrm{mg}$ & NR & $\geq 2$ months & NR & NR & \\
\hline $\begin{array}{l}\text { Torres-Soriano } \\
(2012)^{90}\end{array}$ & $\begin{array}{l}\text { Case series } \\
\text { (prospective) }\end{array}$ & $\begin{array}{l}\text { CNV PDR, RVO } \\
\text { (NR) }\end{array}$ & 31 & NA & $\begin{array}{l}2.5 \mathrm{mg} \text {, frequency not } \\
\text { reported }\end{array}$ & 1.3 & 1 month & NR & NR & \\
\hline $\begin{array}{l}\text { Valmaggia } \\
(2009)^{91}\end{array}$ & $\begin{array}{l}\text { Case series } \\
\text { (retrospective) }\end{array}$ & $\begin{array}{l}\text { CNV due to AMD } \\
\text { (75.5) }\end{array}$ & 324 & NA & $\begin{array}{l}1.25 \mathrm{mg} \text {; then every } \\
6 \text { weeks. Frequency } \\
\text { not reported }\end{array}$ & 3.3 & NR & $\begin{array}{l}\text { Local } \\
\text { pharmacy }\end{array}$ & NR & \\
\hline $\begin{array}{l}\text { Weinberger } \\
(2007)^{92}\end{array}$ & $\begin{array}{l}\text { Case series } \\
\text { (retrospective) }\end{array}$ & $\begin{array}{l}\text { PED in exudative } \\
\text { AMD (76) }\end{array}$ & $31(31)$ & NA & $1.25 \mathrm{mg}$ once & NR & $1-7$ months & NR & Academic institution & \\
\hline $\begin{array}{l}\text { Wickremasinghe } \\
(2008)^{93}\end{array}$ & $\begin{array}{l}\text { Case series } \\
\text { (retrospective) }\end{array}$ & $\begin{array}{l}\text { Neovascular } \\
\text { AMD }\end{array}$ & $\begin{array}{l}1278 \text { IVB } \\
\text { injections }\end{array}$ & NA & $1.25 \mathrm{mg}$ & NR & 1 week & NR & NR & \\
\hline $\mathrm{Wu}(2008)^{94}$ & $\begin{array}{l}\text { interventional case } \\
\text { series (prospective) }\end{array}$ & $\begin{array}{l}\text { Various } \\
\text { (including RVO, } \\
\text { DMO) }\end{array}$ & $1173(1310)$ & NA & $\begin{array}{l}1.25 \mathrm{mg}(16 \%), 2.5 \mathrm{mg} \\
(89 \%)\end{array}$ & $\begin{array}{l}3.7 \text { ( } 3.3 \text { per } \\
\text { eye) }\end{array}$ & $12-15(13.6)$ & NR & No & \\
\hline Yoon $(2012)^{95}$ & $\begin{array}{l}\text { Case series } \\
\text { (retrospective) }\end{array}$ & Myopic CNV (49) & 26 & NA & $1.25 \mathrm{mg}$ & 2.2 & 12 months & NR & NR & $\begin{array}{l}\text { Of the } 40 \text { patients } \\
\text { included in the } \\
\text { study, } 14 \text { received } \\
\text { IVR }\end{array}$ \\
\hline Zhang (2012) ${ }^{96}$ & $\begin{array}{l}\text { Non-randomised } \\
\text { interventional case } \\
\text { series (prospective) }\end{array}$ & $\begin{array}{l}\text { Subfoveal } \\
\text { idiopathic CNV } \\
\text { (32) }\end{array}$ & 40 & NA & $1.25 \mathrm{mg}$ & 2 & 12 months & NR & NR & \\
\hline
\end{tabular}

This table summarises the study characteristics of included observational studies.Data shown here include patient charateristics, interventions and outcomes reported in the included studies.

*Artunay $2009^{35}$ studied patients with the following conditions: AMD, CNV due to myopic degeneration idiopathic and other secondary causes, cystoid or diffuse MO from CRVO, BRVO, diabetes, uveitis and retinitis pigmentosa proliferative retinopathies.

†Population included patients CNV due to AMD, DMO, DR, MO due to RVO or autoimmune retinopathy.

ҒForty-seven patients out of a study population of 71 received bevacizumab. A number of patients received all three anti-VEGF medications while others received just one treatment type. However, authors reported that only the first anti-VEGF injection was considered in the study.

$\S$ Gamulescu $(2010)^{51}$ included a control group that received ranibizumab.

१Re-injection in five eyes, 1 or 2 months after first injection at physician discretion.

${ }^{\star *}$ Good et $a^{\beta 1}$ included a control group that received ranibizumab.

t†101 eyes received bevacizumab only, 96 eyes received ranibizumab only and 18 eyes received bevacizumab and ranibizumab.

¥¥Population included patients AMD, diabetes, retinal vein occlusion and other eye conditions.

$\S \S$ Kiss et $a^{{ }^{6}}$ included a control group that received triamcinolone acetonide.

१ी|Krishnan et $a^{\rho 9}$ included a control group that received ranibizumab.

${ }_{* * *}$ Population included patients with AMD, BRVO, CRVO and myopic choroidal neovascularisation.

†††Population included patients with neovascular AMD; BRVO, CRVO; cystoid macular oedema; proliferative DR and DMO.

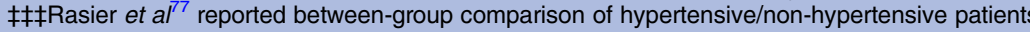

$\S \S \S$ Conditions included AMD, DR, CNV, BRVO, CRVO and other pathologies (unspecified).

११११Control group received triamcinolone acetonide.

AMD, age-related macular degeneration; BP, blood pressure; BRVO, branch retinal vein occlusion; CRVO, central retinal vein occlusion; CMO, cystoid macular oedema; CNV, choroidal neovascularisation; DMO, diabetic macular oedema; DR, diabetic retinopathy; FA, fluorescein angiography; IVB, intravitreal bevacizumab; IVP, intravitreal pegaptinib; IVR, intravitreal ranibizumab; MO, macular oedema; NA, not applicable, NR, not

reported; OCT, optical coherence tomography; PDT, photodynamic therapy; RVO, retinal vein occlusion; PED, pigment epithelium detachment. 


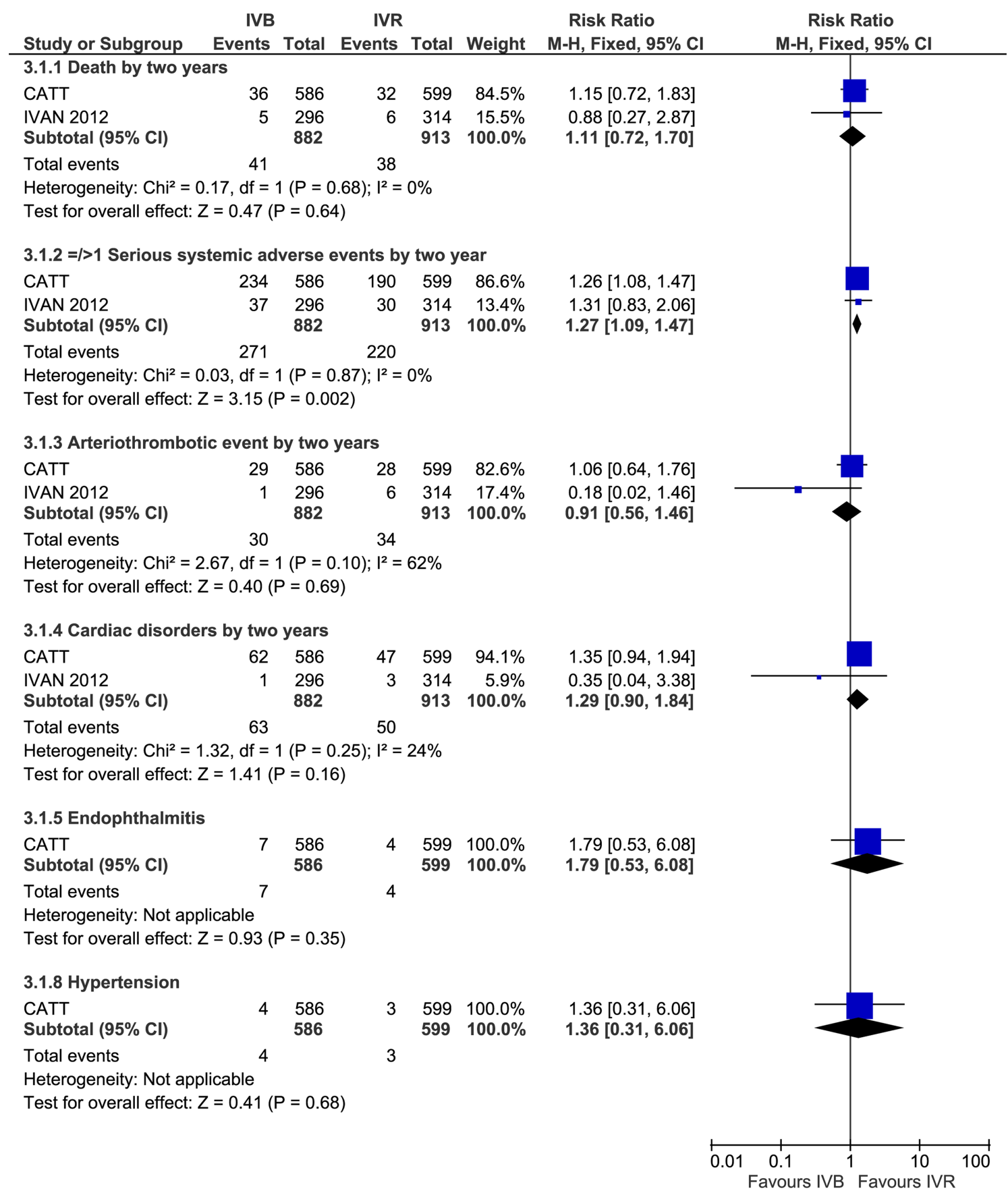

Figure 2 Pooled analysis of systemic adverse events comparing intravitreal bevacizumab with intravitreal ranibizumab in patients with age-related macular degeneration. This figure shows the pooled effect estimate for systemic adverse events comparing intravitreal bevacizumab with intravitreal ranibizumab in patients with age-related macular degeneration. IVB, intravitreal bevacizumab; IVR, intravitreal ranibizumab. This figure has been reproduced from the full report related to this project available at http://www.nicedsu.org.uk/Bevacizumab\%20report\%20\%20NICE\%20published\%20version\%2011.04.13.pdf.

inadequate follow-up periods (less than 6 months). With respect to larger studies, observational data from Curtis et $a l^{45}$ suggest no difference in the risk of AEs between IVB and IVR once socioeconomic confounders are accounted for. On the other hand, results of an unpublished study of Medicare patients funded by Genentech ${ }^{54}$ found an increased risk of stroke and death in IVB patients. The available abstract, however, did not provide sufficient information to allow an in-depth analysis of the results of this study. A recently published population-based, nested case-control study reported by Campbell et a ${ }^{{ }^{8}}(\mathrm{n}=91378)$ found no relationship between the risk of MI, venous thromboembolism, stroke or congestive heart failure and the administration of IVR or IVB. While the risk of systemic AEs was similar for both treatment groups, there was an increased risk of acute MI for a subgroup of patients with diabetes who received IVB. 
Table 3 Serious systemic and ocular adverse events reported in included observational studies

\begin{tabular}{|c|c|c|c|}
\hline \multicolumn{4}{|c|}{ Systemic adverse events } \\
\hline Adverse event & Rates (\%) & $\begin{array}{l}\text { Number of eligible } \\
\text { studies contributing } \\
\text { data }\end{array}$ & Percentage of eligible studies reporting zero events \\
\hline Death & 0.4 to 3.8 & $6^{*}$ & Not applicable \\
\hline Hospitalisation & $32 \dagger$ & 1 & Not applicable \\
\hline $\begin{array}{l}\text { Non-ocular } \\
\text { haemorrhage }\end{array}$ & 0.0 & 1 & $100 \%(n=1)^{82}$ \\
\hline $\begin{array}{l}\text { Arterial } \\
\text { thromboembolism }\end{array}$ & 0.0 to $1.35 \ddagger$ & 9 & $78 \%(n=7)^{34384482858896}$ \\
\hline Hypertension & 0.0 to 15.6 & 9 & $44 \%(n=4) 42438296$ \\
\hline $\begin{array}{l}\text { Myocardial } \\
\text { infarction }\end{array}$ & 0.0 to 8.2 & 10 & $50 \%(n=5)^{3438566782}$ \\
\hline $\begin{array}{l}\text { Cerebrovascular } \\
\text { accident }\end{array}$ & 0.0 to 8.7 & $11 \S$ & $45 \%(n=5)^{3438566782}$ \\
\hline $\begin{array}{l}\text { Transient ischaemic } \\
\text { attack }\end{array}$ & 0.4 to 1.0 & $5^{34}$ & $60 \%(n=3)^{34} 6782$ \\
\hline \multicolumn{4}{|l|}{ Ocular adverse events } \\
\hline $\begin{array}{l}\text { Infectious } \\
\text { endophthalmitis }\end{array}$ & 0.0 to 1.0 & $31 \rrbracket$ & $\begin{array}{l}62 \% \\
(n=19)^{29} 324143445156596065677376818586889196\end{array}$ \\
\hline Retinal detachment & 0.0 to 29.0 & 20 & $75 \%(n=15)^{29} 3235566567687679818586919596$ \\
\hline Retinal tear & 0.0 to 15.0 & 14 & $42 \%(n=6)^{29} 4452606585$ \\
\hline $\begin{array}{l}\text { Anterior chamber } \\
\text { reaction }\end{array}$ & 0.0 to 50.0 & $21^{\star \star}$ & $57 \%(n=12)^{29} 4143445156676885889196$ \\
\hline $\begin{array}{l}\text { Ocular } \\
\text { haemorrhage }\end{array}$ & 0.0 to 72.0 & 14 & $43 \%(n=6)^{32} 4152568591$ \\
\hline Lens damage & 0.0 to 0.5 & 9 & $67 \%(n=6)^{415665678591}$ \\
\hline Ocular hypertension & 0.0 to 20.0 & $16 \dagger \dagger$ & $50 \%(n=8)^{29} 32364165708791$ \\
\hline Visual loss & 0.0 to 50.0 & 9 & $11 \%(n=1)^{85}$ \\
\hline \multicolumn{4}{|c|}{$\begin{array}{l}\text { Estimates of adverse event incidence were calculated by dividing the number of reported events by the number of patients that received IVB } \\
\text { (event rate per patient) or the number of eyes treated (event rate per treated eye). } \\
\text { *One study presented an HR of } 1.11(99 \% \mathrm{Cl} 1.01 \text { to } 1.23 \text {, IVB vs IVR). } \\
\text { tIncidence of systemic adverse events was reported based on number of injections and not patients, } 32 \% \text { ( } \mathrm{n}=222 / 693 \text { injections). } \\
\text { fEvent rate was presented for a sub-group to the study population living in a specified geographical area. } \\
\S \text { One study reported an HR of } 1.57(99 \% \mathrm{Cl} 1.04 \text { to } 2.37 \text {, IVB vs IVR). } \\
\text { TAuthors of one study stated that the rate of infectious endophthalmitis after an IVB injection of } 1.5 \text { mg may be approximately } 1: 1000 \text {. } \\
\star_{*} \text { One study reported an HR of } 1.8(99 \% \mathrm{Cl} 1.2 \text { to } 2.8 \text {, IVB vs IVR). } \\
\text { ††One study reported an HR of } 0.81(99 \% \mathrm{Cl} 0.71 \text { to } 0.93 \text {, IVB vs IVR). }\end{array}$} \\
\hline
\end{tabular}

This review highlighted the challenges of assessing the safety of IVB especially due to limited opportunities for in-depth detailed analyses of the relationship between IVB preparation and reported rates of infectious endophthalmitis. In the past, case reports have suggested contaminated batches of IVB as the primary source of infection; a published review of patient safety information held by the National Patient Safety Agency in England and Wales ${ }^{99}$ reported an increased risk of serious AEs including endophthalmitis following IVB treatment. The authors acknowledged that identifying the source of infection (ie, contaminated injection procedure or infected anti-VEGF) could be complex. However, Jonas et $a l,{ }^{63}$ reporting on $\mathrm{AE}$ rates in a study population which included patients who had received IVB and IVT, suggested that event rates were statistically independent of drug injected $(\mathrm{p}=0.45)$, operating surgeon $(p=0.18)$ and patient's age $(p=0.87)$.

It is also important to highlight limitations of this review. By relying on the previous systematic review ${ }^{5}$ as a source of evidence, it is possible that studies that were not identified in that review may have been missed in this review. Our searches were undertaken up to 2012. An updated electronic literature search was conducted up to 23 May 2014, retrieving a total of 1300 records. A preliminary shift of titles resulted in 333 potentially relevant abstracts for further detailed examination. We would prefer to have undertaken a full update. Unfortunately, this is not possible for us at present due to lack of the extensive time and resources required. Although comprehensive and up-to-date systematic reviews are desirable, a recent analysis of a sample of systematic reviews showed that the median duration of survival indicating a requirement for an update was 5.5 years (95\% CI 4.36 to 7.67$)$ in systematic reviews of randomised trials of procedures or conventional drugs. ${ }^{100}$ Furthermore, many RCTs randomised small numbers of participants and these may have been underpowered to detect differences in AEs. ${ }^{4} 97$ Generalisability of findings may also be limited due to 
differences between study participants and patients seen in routine practice. In addition, there were concerns relating to ascertainment of exposure particularly in observational studies. ${ }^{98}$ The influence of excluding non-English publications in this review is unclear. Additionally, adopting a narrow focus in the definition of AEs implies that data on less serious or rare events were not presented.

\section{CONCLUSIONS}

Overall, rates of serious AEs following IVB were low when compared to other intravitreal treatments, sham injection and laser therapy with relatively higher rates being reported in head-to-head studies of IVB versus ranibizumab. Most outcomes were, however, not significantly different between treatment groups. Current evidence from observational data still remains limited due to relatively small sample sizes, unclear definition, evaluation and reporting of safety outcomes as well as adequate follow-up periods. However, an opportunity to explore the relationship between the incidence of AEs and other variables such as injection techniques, pre-existing risk factors (eg, immunosuppression, cross-contamination) and quality of IVB could offer cost-saving options in providing treatment for certain ophthalmic conditions.

Acknowledgements We would like to thank Jennifer Dunn for administrative support throughout the project.

Contributors EP coordinated the systematic review and was responsible for the conception and design, acquisition of data, analysis and interpretation of data and drafting and revision of the final manuscript. JR was responsible for the conception and design, acquisition of data, analysis and interpretation of data and drafting and revision of the final manuscript. RW was responsible for the developing and undertaking the electronic literature searches and drafting and revision of the final manuscript. $\mathrm{EE}-\mathrm{H}$ and $\mathrm{ME}$ were responsible for acquisition of data, analysis and interpretation of data and drafting and revision of the final manuscript. AP contributed to the conception and design of the study, assisted with data collection analysis, interpretation of data and drafting and revision of the final manuscript. AW contributed to the conception and design of the study, assisted with analysis and interpretation of data and drafting and revision of the final manuscript.

Funding This manuscript is based on a report which was funded by the National Institute for Health and Care Excellence ('NICE') through its Decision Support Unit.

Competing interests None.

Provenance and peer review Not commissioned; externally peer reviewed.

Data sharing statement Extra data relating to references of excluded full-text articles is available by emailing Edith Poku (e.poku@sheffield.ac.uk).

Open Access This is an Open Access article distributed in accordance with the Creative Commons Attribution Non Commercial (CC BY-NC 4.0) license, which permits others to distribute, remix, adapt, build upon this work noncommercially, and license their derivative works on different terms, provided the original work is properly cited and the use is non-commercial. See: http:// creativecommons.org/licenses/by-nc/4.0/

\section{REFERENCES}

1. Bunce $C$, Wormald $R$. Leading causes of certification for blindness and partial sight in England and Wales. BMC Public Health 2006;6:58.

2. Soheilian M, Garfami KH, Ramezani A, et al. Two-year results of a randomized trial of intravitreal bevacizumab alone or combined with triamcinolone versus laser in diabetic macular edema. Retina 2012;32:314-21.

3. Raftery J, Clegg A, Jones J, et al. Ranibizumab (Lucentis) versus bevacizumab (Avastin): modelling cost effectiveness. $\mathrm{Br} \mathrm{J}$ Opthalmol 2007;91:1244-6.

4. Schmucker C, Loke YK, Ehlken C, et al. Intravitreal bevacizumab (Avastin) versus ranibizumab (Lucentis) for the treatment of age-related macular degeneration: a safety review. Br J Opthalmol 2011;95:308-17.

5. van der Reis MI, La Heij EC, De Jong-Hesse Y, et al. A systematic review of the adverse events of intravitreal anti-vascular endothelial growth factor injections. Retina 2011;31:1449-69.

6. Downs $\mathrm{SH}$, Black $\mathrm{N}$. The feasibility of creating a checklist for the assessment of the methodological quality both of randomised and non-randomised studies of health care interventions. J Epidemiol Community Health 1998;52:377-84

7. Ahmadieh $\mathrm{H}$, Ramezani A, Shoeibi $\mathrm{N}$, et al. Intravitreal bevacizumab with or without triamcinolone for refractory diabetic macular edema; a placebo-controlled, randomized clinical trial. Graefes Arch Clin Exp Ophthalmol 2008;246:483-9.

8. Cekic O, Cakir M, Yazici AT, et al. A comparison of three different intravitreal treatment modalities of macular edema due to branch retinal vein occlusion. Curr Eye Res 2010;35:925-9.

9. Michaelides $M$, Kaines A, Hamilton RD, et al. A prospective randomized trial of intravitreal bevacizumab or laser therapy in the management of diabetic macular edema (BOLT study) 12-month data: report 2. Ophthalmology 2010;117:1078-86.

10. Scott IU, Edwards AR, Beck RW, et al.; Diabetic Retinopathy Clinical Research Network. A phase 2 randomized clinical trial of intravitreal bevacizumab for diabetic macular edema. Ophthalmology 2007;114:1860-7.

11. Faghihi H, Roohipoor R, Mohammadi SF, et al. Intravitreal bevacizumab versus combined bevacizumab-triamcinolone versus macular laser photocoagulation in diabetic macular edema. Eur $J$ Ophthalmol 2008;18:941-8.

12. Epstein DLJ, Algvere PV, von Wendt G, et al. Bevacizumab for macular edema in central retinal vein occlusion: a prospective, randomized, double-masked clinical study. Ophthalmology 2012:119:1184-9.

13. Moradian S, Faghihi $\mathrm{H}$, Sadeghi B, et al. Intravitreal bevacizumab vs. sham treatment in acute branch retinal vein occlusion with macular edema: results at 3 months. Graefes Arch Clin Exp Ophthalmol 2011;249:193-200.

14. Bashshur ZF, Schakal A, Hamam RN, et al Intravitreal bevacizumab vs verteporfin photodynamic therapy for neovascular age-related macular degeneration. Arch Ophthalmol 2007;125:1357-61.

15. Biswas P, Sengupta S, Choudhary R, et al. Comparing ranibizumab with bevacizumab. Ophthalmology 2011 118:600-600.e2. http://www.sciencedirect.com/science/article/pii/ S0161642010011036 (accessed 10 Jun 2012).

16. Martin DF, Maguire MG, Fine SL, et al:; CATT Research Group. Ranibizumab and bevacizumab for treatment of neovascular age-related macular degeneration: two-year results. Ophthalmology 2012;119:1388-98.

17. Ding X, Li J, Hu X, et al. Prospective study of intravitreal triamcinolone acetonide versus bevacizumab for macular edema secondary to central retinal vein occlusion. Retina 2011 ; 31:838-45

18. Gharbiya M, Giustolisi R, Allievi F, et al. Choroidal neovascularization in pathologic myopia: intravitreal ranibizumab versus bevacizumab-a randomized controlled trial. $\mathrm{Am} \mathrm{J}$ Ophthalmol 2010;149:458-64.

19. Chakravarthy U, Harding SP, Rogers CA, et al. Ranibizumab versus bevacizumab to treat neovascular age-related macular degeneration: one-year findings from the IVAN Randomized Trial. Ophthalmology 2012;119:1399-411.

20. Lazic R, Gabric N. Verteporfin therapy and intravitreal bevacizumab combined and alone in choroidal neovascularization due to age-related macular degeneration. Ophthalmology 2007;114:1179-85.

21. Lim JW, Lee HK, Shin MC. Comparison of intravitreal bevacizumab alone or combined with triamcinolone versus triamcinolone in diabetic macular edema: a randomized clinical trial. Ophthalmologica 2012;227:100-6.

22. Marey HM, Ellakwa AF. Intravitreal bevacizumab alone or combined with triamcinolone acetonide as the primary treatment for diabetic macular edema. Clin Ophthalmol 2011;5:1011-16.

23. Patwardhan SD, Azad R, Shah BM, et al. Role of intravitreal bevacizumab in Eales disease with dense vitreous hemorrhage: a prospective randomized control study. Retina 2011;31:866-70. 
24. Schmid-Kubista KE, Krebs I, Ansari-Shahrezaei S, et al. Comparing treatment of neovascular age-related macular degeneration with sequential intravitreal Avastin and Macugen versus intravitreal mono-therapy-a pilot study. Curr Eye Res 2011;36:958-63.

25. Shahin MM, El-Lakkany RS, Shahin MM, et al. A prospective, randomized comparison of intravitreal triamcinolone acetonide versus intravitreal bevacizumab (avastin) in diffuse diabetic macular edema. Middle East Afr J Ophthalmol 2010;17:250-3.

26. Tufail A, Patel PJ, Egan C, et al. Bevacizumab for neovascular age related macular degeneration ( $\mathrm{ABC}$ Trial): multicentre randomised double masked study. BMJ 2010;340:c459.

27. Yazdani S, Hendi K, Pakravan M, et al. Intravitrealbevacizumab for neovascular glaucoma: a randomized controlled trial. J Glaucoma 2009;18:632-7.

28. Bashshur ZF, Haddad ZA, Schakal AR, et al. Intravitreal bevacizumab for treatment of neovascular age-related macular degeneration: the second year of a prospective study. $A m \mathrm{~J}$ Ophthalmol 2009;148:59-65.

29. Kim KS, Jee D. Effect of the Honan intraocular pressure reducer on intraocular pressure increase following intravitreal injection using the tunneled scleral technique. Jpn J Ophthalmol 2011;55:632-7.

30. Arias L, Planas N, Prades S, et al. Intravitreal bevacizumab (Avastin) for choroidal neovascularisation secondary to pathological myopia: 6-month results. $\mathrm{Br} J$ Ophthalmol 2008;92:1035-9.

31. Good TJ, Kimura AE, Mandava N, et al. Sustained elevation of intraocular pressure after intravitreal injections of anti-VEGF agents. Br J Ophthalmol 2011;95:1111-14.

32. Kim JY, Park SP. Comparison between intravitreal bevacizumab and triamcinolone for macular edema secondary to branch retina vein occlusion. Korean J Ophthalmol 2009;23:259-65.

33. Abraham-Marin ML, Cortes-Luna CF, Alvarez-Rivera G, et al. Intravitreal bevacizumab therapy for neovascular age-related macular degeneration: a pilot study. Graefes Arch Clin Exp Ophthalmol 2007;245:651-5.

34. Arevalo JF, Sanchez JG, Wu L, et al. Intravitreal bevacizumab for subfoveal choroidal neovascularization in age-related macular degeneration at twenty-four months: the Pan-American Collaborative Retina Study. Ophthalmology 2010;117:1974-81.

35. Artunay O, Yuzbasioglu E, Rasier R, et al. Incidence and management of acute endophthalmitis after intravitreal bevacizumab (Avastin) injection. Eye 2009;23:2187-93.

36. Azad RV, Khan MA, Chanana B, et al. Intravitreal bevacizumab for subfoveal choroidal neovascularization secondary to age-related macular degeneration in an Indian population. Jpn J Ophthalmol 2008;52:52-6.

37. Baba T, Kubota-Taniai M, Kitahashi M, et al. Two-year comparison of photodynamic therapy and intravitreal bevacizumab for treatment of myopic choroidal neovascularisation. $\mathrm{Br} \mathrm{J}$ Opthalmol 2010;94:864-70

38. Bakri SJ, Risco M, Edwards AO, et al. Bilateral simultaneous intravitreal injections in the office setting. Am J Ophthalmol 2009;148:66-9.

39. Carneiro AM, Falcao MS, Brandao EM, et al. Intravitreal bevacizumab for neovascular age-related macular degeneration with or without prior treatment with photodynamic therapy: one-year results. Retina 2010;30:85-92.

40. Carneiro AM, Barthelmes D, Falcao MS, et al. Arterial thromboembolic events in patients with exudative age-related macular degeneration treated with intravitreal bevacizumab or ranibizumab. Ophthalmologica 2011;225:211-21.

41. Chen $\mathrm{CH}$, Chen $\mathrm{YH}, \mathrm{Wu} \mathrm{PC}$, et al. Treatment of branch retinal vein occlusion induced macular edema in treatment-naive cases with a single intravitreal triamcinolone or bevacizumab injection. Chang Gung Med J 2010;33:424-35

42. Cleary CA, Jungkim S, Ravikumar K, et al. Intravitreal bevacizumab in the treatment of neovascular age-related macular degeneration, 6-and 9-month results. Eye 2008;22:82-6.

43. Costa RA, Jorge R, Calucci D, et al. Intravitreal bevacizumab for choroidal neovascularization caused by AMD (IBeNA Study): results of a phase 1 dose-escalation study. Invest Ophthalmol Vis Sci 2006;47:4569-78.

44. Costagliola C, Romano M, Corte MD, et al. Intravitreal bevacizumab for treatment-naive patients with subfoveal occult choroidal neovascularization secondary to age-related macular degeneration: a 12-month follow-up study. Retina 2009:29:1227-34.

45. Curtis LH, Hammill BG, Schulman KA, et al. Risks of mortality, myocardial infarction, bleeding, and stroke associated with therapies for age-related macular degeneration. Arch Ophthalmol 2010;128:1273-79.
46. Falkenstein I, Cheng L, Freeman WR. Changes of intraocular pressure after intravitreal injection of bevacizumab (Avastin). Retina 2007;27:1044-7.

47. Fintak DR, Shah GK, Blinder KJ, et al. Incidence of endophthalmitis related to intravitreal injection of bevacizumab and ranibizumab. Retina 2008;28:1395-9.

48. Fong KCS, Kirkpatrick N, Mohamed Q, et al. Intravitreal bevacizumab (Avastin) for neovascular age-related macular degeneration using a variable frequency regimen in eyes with no previous treatment. Clin Experiment Ophthalmol 2008;36:748-55.

49. Frenkel MP, Haji SA, Frenkel RE. Effect of prophylactic intraocula pressure-lowering medication on intraocular pressure spikes after intravitreal injections. Arch Ophthalmol 2010;128:1523-7.

50. Fukami $T$, Kitahashi M Sato $E$, et al Six cases of sterile endophthalmitis developed consecutively after intravitreal injection of bevacizumab [abstract]. Nippon Ganka Gakkai Zasshi-Acta Societatis Ophthalmologicae Japonicae 2011;115:706-10.

51. Gamulescu MA, Radeck V, Lustinger B, et al. Bevacizumab versus ranibizumab in the treatment of exudative age-related macular degeneration. Int Ophthalmol 2010;30:261-6.

52. Gomi F, Sawa M, Sakaguchi $\mathrm{H}$, et al. Efficacy of intravitreal bevacizumab for polypoidal choroidal vasculopathy. $\mathrm{Br} J$ Opthalmol 2008;92:70-3.

53. Goverdhan SV, Lochhead J. Submacular haemorrhages after intravitreal bevacizumab for large occult choroidal neovascularisation in age-related macular degeneration. $\mathrm{Br} \mathrm{J}$ Opthalmol 2008;92:210-12.

54. Gower EW, Cassard S, Chu L, et al. Adverse event rates following intravitreal injection of avastin or lucentis for treating age-related macular degeneration [abstract]. Invest Ophthalmol Vis Sci 2011;129; ARVO Suppl.

55. Hernandez-Rojas ML, Quiroz-Mercado H, Dalma-Weiszhausz J, et al. Short-term effects of intravitreal bevacizumab for subfoveal choroidal neovascularization in pathologic myopia. Retina 2007;27:707-12

56. Higashide T, Murotani E, Saito Y, et al. Adverse events associated with intraocular injections of bevacizumab in eyes with neovascular glaucoma. Graefes Arch Clin Exp Ophthalmol 2012;250:603-10.

57. Hollands $\mathrm{H}$, Wong J, Bruen R, et al Short-term intraocular pressure changes after intravitreal injection of bevacizumab. Can $J$ Ophthalmol 2007;42:807-11.

58. Ikuno Y, Sayanagi K, Soga K, et al. Intravitreal bevacizumab for choroidal neovascularization attributable to pathological myopia: one-year results. Am J Ophthalmol 2009;147:94-100.

59. Inman ZD, Anderson NG. Incidence of endophthalmitis after intravitreal injection of antivascular endothelial growth factor medications using topical lidocaine gel anesthesia. Retina 2011;31:669-72.

60. Jaissle GB, Leitritz M, Gelisken F, et al. One-year results after intravitreal bevacizumab therapy for macular edema secondary to branch retinal vein occlusion. Graefes Arch Clin Exp Ophthalmol 2009;247:27-33.

61. Johnson D, Hollands $\mathrm{H}$, Hollands $\mathrm{S}$, et al. Incidence and characteristics of acute intraocular inflammation after intravitreal injection of bevacizumab: a retrospective cohort study. Can $J$ Ophthalmol 2010;45:239-42.

62. Jonas JB, Spandau UH, Rensch F, et al. Infectious and noninfectious endophthalmitis after intravitreal bevacizumab. J Ocul Pharmacol Ther 2007;23:240-2

63. Jonas JB, Spandau UH, Schlichtenbrede F. Short-term complications of intravitreal injections of triamcinolone and bevacizumab. Eye 2008;22:590-1.

64. Julian K, Terrada C, Fardeau C, et al. Intravitreal bevacizumab as first local treatment for uveitis-related choroidal neovascularization: long-term results. Acta Opthalmol 2011;89:179-84.

65. Kim M, Lee $\mathrm{P}, \mathrm{Kim} \mathrm{Y}$, et al. Effect of intravitreal bevacizumab based on optical coherence tomography patterns of diabetic macular edema. Ophthalmologica 2011;226:138-44.

66. Kiss $\mathrm{C}$, Michels S, Prager F, et al. Evaluation of anterior chamber inflammatory activity in eyes treated with intravitreal bevacizumab. Retina 2006;26:877-81.

67. Krebs I, Lie S, Stolba U, et al. Efficacy of intravitreal bevacizumab (Avastin) therapy for early and advanced neovascular age-related macular degeneration. Acta Opthalmol 2009;87:611-17.

68. Kriechbaum K, Michels S, Prager F, et al. Intravitreal Avastin for macular oedema secondary to retinal vein occlusion: a prospective study. Br J Ophthalmol 2008;92:518-22.

69. Krishnan R, Goverdhan S, Lochhead J, et al. Submacular haemorrhage after intravitreal bevacizumab compared with intravitreal ranibizumab in large occult choroidal neovascularization. Clin Experiment Ophthalmol 2009;37:384-8. 
70. Kumar A, Sehra SV, Thirumalesh MB, et al. Secondary rhegmatogenous retinal detachment following intravitreal bevacizumab in patients with vitreous hemorrhage or tractional retinal detachment secondary to Eales' disease. Graefes Arch Clin Exp Ophthalmol 2012;250:685-90.

71. Lazic R, Gabric N. Intravitreally administered bevacizumab (Avastin) in minimally classic and occult choroidal neovascularization secondary to age-related macular degeneration. Graefes Arch Clin Exp Ophthalmol 2007;245:68-73.

72. Lima LH, Zweifel SA, Engelbert M, et al. Evaluation of safety for bilateral same-day intravitreal injections of antivascular endothelial growth factor therapy. Retina 2009;29:1213-17.

73. Lommatzsch A, Heimes B, Gutfleisch M, et al. Serous pigment epithelial detachment in age-related macular degeneration: comparison of different treatments. Eye 2009;23:2163-8.

74. Lorenz K, Zwiener I, Mirshahi A. Subconjunctival reflux and need for paracentesis after intravitreal injection of $0.1 \mathrm{ml}$ bevacizumab: comparison between 27-gauge and 30-gauge needle. Graefes Arch Clin Exp Ophthalmol 2010;248:1573-7.

75. Mason JO, White MF, Feist RM, et al. Incidence of acute onset endophthalmitis following intravitreal bevacizumab (Avastin) injection. Retina 2008;28:564-7.

76. Manayath GJ, Narendran V, Al-Kharousi N, et al. Bevacizumab therapy for macular edema in central retinal vein occlusion: Long-term results. Oman J Ophthalmol 2009;2:73-8.

77. Rasier R, Artunay O, Yuzbasioglu E, et al. The effect of intravitrea bevacizumab (avastin) administration on systemic hypertension. Eye 2009;23:1714-18.

78. Russo V, Barone A, Conte E, et al. Bevacizumab compared with macular laser grid photocoagulation for cystoid macular edema in branch retinal vein occlusion. Retina 2009;29:511-15.

79. Saeed MU, Qureshi F, Batra R, et al. Effect of reflux of drug during intravitreal anti-VEGF therapies on foveal thickness. Semin Ophthalmol 2011;26:61-3.

80. Shah CP, Garg SJ, Vander JF, et al. Outcomes and risk factors associated with endophthalmitis after intravitreal injection of anti-vascular endothelial growth factor agents. Ophthalmology 2011;118:2028-34.

81. Sharma S, Johnson D, Abouammoh M, et al. Rate of serious adverse effects in a series of bevacizumab and ranibizumab injections. Can J Ophthalmol 2012;47:275-9.

82. Shienbaum G, Gupta OP, Fecarotta C, et al. Bevacizumab for neovascular age-related macular degeneration using a treat-andextend regimen: clinical and economic impact. Am J Opthalmol 2012;153:468-73.

83. Shima C, Sakaguchi $\mathrm{H}$, Gomi F, et al. Complications in patients after intravitreal injection of bevacizumab. Acta Ophthalmol 2008;86:372-6.

84. Shimada N, Ohno-Matsui K, Hayashi K, et al. Macular detachment after successful intravitreal bevacizumab for myopic choroidal neovascularization. Jpn J Ophthalmol 2011;55:378-82.

85. Sivkova N, Koleva-Georgieva D. Bevacizumab for the treatment of macular oedema in patients with diabetic retinopathy and retinal vascular occlusive disorders. Auton Autacoid Pharmacol 2010;30:144-7.
86. Sohn $\mathrm{HJ}$, Han $\mathrm{DH}$, Kim IT, et al. Changes in aqueous concentrations of various cytokines after intravitreal triamcinolone versus bevacizumab for diabetic macular edema. Am J Opthalmol 2011;152:686-94.

87. Song JH, Lee JJ, Lee SJ, et al. Comparison of the short-term effects of intravitreal triamcinolone acetonide and bevacizumab injection for diabetic macular edema. Korean $J$ Ophthalmol 2011;25:156-60.

88. Sonmez K, Sonmez PA, Ozkan SS, et al. One-year outcomes of less frequent bevacizumab in age-related macular degeneration. Retina 2011;31:645-53.

89. Spandau UHM, Jonas JB. Retinal pigment epithelium tear after intravitreal bevacizumab for exudative age-related macular degeneration. Am J Opthalmol 2006;142:1068-70.

90. Torres-Soriano ME, Cubas-Lorenzo V, Garcia-Aguirre G, et al. Multifocal electrophysiologic findings after intravitreal bevacizumab (avastin) treatment. Retina 2012;32:972-6.

91. Valmaggia $\mathrm{C}$, Haueter I, Kloos $\mathrm{P}$, et al. The treatment of choroida neovascularizations in age-related macular degeneration using either avastin or lucentis. Klin Monbl Augenheilkd 2009; 226:294-8.

92. Weinberger AWA, Thiel M, Mohammadi B, et al. Retinal pigment epithelium tears after intravitreal bevacizumab in pigment epithelium detachment. Am J Opthalmol 2007;144:294-6.

93. Wickremasinghe SS, Michalova K, Gilhotra J, et al. Acute intraocular inflammation after intravitreous injections of bevacizumab for treatment of neovascular age-related macular degeneration. Ophthalmology 2008;115:1911-15.

94. Wu L, Martinez-Castellanos MA, Quiroz-Mercado H, et al. Twelve-month safety of intravitreal injections of bevacizumab (Avastin): results of the Pan-American Collaborative Retina Study Group (PACORES). Graefes Arch Clin Exp Ophthalmol 2008;246:81-7.

95. Yoon JU, Kim YM, Lee SJ, et al. Prognostic factors for visual outcome after intravitreal anti-vegf injection for naive myopic choroidal neovascularization. Retina 2012;32:949-55.

96. Zhang H, Liu ZL, Sun $\mathrm{P}$, et al. Intravitreal bevacizumab for treatment of subfoveal idiopathic choroidal neovascularization results of a 1-year prospective trial. Am J Opthalmol 2012;153:300-6.

97. Chakravarthy U, Harding SP, Rogers CA, et al. Alternative treatments to inhibit VEGF in age-related choroidal neovascularisation: 2-year findings of the IVAN randomised controlled trial. Lancet 2013;382:1258-67.

98. Campbell RJ, Sudeep SG, Bronskill SE, et al. Adverse events with intravitreal injection of vascular endothelial growth factor inhibitors: nested case-control study. BMJ 2012;345:e4203.

99. Kelly SP, Barua A. A review of safety incidents in England and Wales for vascular endothelial growth factor inhibitor medications. Eye 2011;25:710-16.

100. Shojania KG, Sampson M, Ansari MT, et al. How quickly do systematic reviews go out of date? Ann Intern Med 2007;147:224-33. 